\title{
Functional Regeneration of Supraspinal Connections in a Patient With Transected Spinal Cord Following Transplantation of Bulbar Olfactory Ensheathing Cells With Peripheral Nerve Bridging
}

\author{
Pawel Tabakow,* Geoffrey Raisman, $\dagger$ Wojciech Fortuna, $* \ddagger$ Marcin Czyz,* Juliusz Huber, $\S$ Daqing Li, $\dagger$ \\ Pawel Szewczyk, II Stefan Okurowski,\# Ryszard Miedzybrodzki, ${ }^{*} * \dagger \dagger$ Bogdan Czapiga, ${ }^{*}$ Beata Salomon, \\ Agnieszka Halon,§§ Ying Li,† Joanna Lipiec,§ Aleksandra Kulczyk, § and Wlodzimierz Jarmundowicz* \\ *Department of Neurosurgery, Wroclaw Medical University, Wroclaw, Poland \\ $†$ Spinal Repair Unit, Department of Brain Repair and Rehabilitation, UCL Institute of Neurology, London, UK \\ †Ludwik Hirszfeld Institute of Immunology and Experimental Therapy, Polish Academy of Sciences, Wroclaw, Poland \\ $\S$ Department of Pathophysiology of Locomotor Organs, Karol Marcinkowski Medical University, Poznan, Poland \\ ID Department of General Radiology, Interventional Radiology and Neuroradiology, Wroclaw Medical University, Wroclaw, Poland \\ \#Neurorehabilitation Center for Treatment of Spinal Cord Injuries AKSON, Wroclaw, Poland \\ **Bacteriophage Laboratory of the Ludwik Hirszfeld Institute of Immunology, and Experimental Therapy, \\ Polish Academy of Sciences, Wroclaw, Poland \\ $\dagger †$ Department of Clinical Immunology of the Transplantation Institute, Medical University of Warsaw, Warsaw, Poland \\ $\$ \neq$ University Clinical Hospital, Wroclaw, Poland \\ $\S \S$ Department of Pathomorphology and Oncological Cytology, Wroclaw Medical University, Wroclaw, Poland
}

\begin{abstract}
Treatment of patients sustaining a complete spinal cord injury remains an unsolved clinical problem because of the lack of spontaneous regeneration of injured central axons. A 38-year-old man sustained traumatic transection of the thoracic spinal cord at upper vertebral level Th9. At 21 months after injury, the patient presented symptoms of a clinically complete spinal cord injury (American Spinal Injury Association class A-ASIA A). One of the patient's olfactory bulbs was removed and used to derive a culture containing olfactory ensheathing cells and olfactory nerve fibroblasts. Following resection of the glial scar, the cultured cells were transplanted into the spinal cord stumps above and below the injury and the 8-mm gap bridged by four strips of autologous sural nerve. The patient underwent an intense pre- and postoperative neurorehabilitation program. No adverse effects were seen at 19 months postoperatively, and unexpectedly, the removal of the olfactory bulb did not lead to persistent unilateral anosmia. The patient improved from ASIA A to ASIA C. There was improved trunk stability, partial recovery of the voluntary movements of the lower extremities, and an increase of the muscle mass in the left thigh, as well as partial recovery of superficial and deep sensation. There was also some indication of improved visceral sensation and improved vascular autoregulation in the left lower limb. The pattern of recovery suggests functional regeneration of both efferent and afferent long-distance fibers. Imaging confirmed that the grafts had bridged the left side of the spinal cord, where the majority of the nerve grafts were implanted, and neurophysiological examinations confirmed the restitution of the integrity of the corticospinal tracts and the voluntary character of recorded muscle contractions. To our knowledge, this is the first clinical indication of the beneficial effects of transplanted autologous bulbar cells.
\end{abstract}

Key words: Paraplegia; Cell transplantation; Repair; Regeneration

\section{INTRODUCTION}

Olfactory ensheathing cells (OECs) are a population of glial cells residing both in the peripheral and central nervous systems. Together with their accompanying envelope of olfactory nerve fibroblasts (ONFs), they enfold the bundles of olfactory nerve fibers in their course from the nasal mucosa to make synaptic connections in the olfactory bulb (OB) $(9,28,34,36)$. Animal experiments have shown that transplantation of OECs and ONFs cultured from the $\mathrm{OB}$ mediate regeneration and functional reconnection of severed axons in spinal cord and brachial plexus injuries $(20,22,23,35,37)$, whereas cells cultured

Received February 18, 2014; final acceptance September 8, 2014. Online prepub date: October 21, 2014.

Address correspondence to Pawel Tabakow, M.D., Ph.D., Department of Neurosurgery, Wroclaw Medical University, Borowska str. 213, 50-556 Wroclaw, Poland. Tel: +48 606137 846; Fax: +48 7173434 09; E-mail: p.tabakov@wp.pl 
from the olfactory mucosa have only minor benefits in corticospinal tract injuries (46) and do not appear to mediate regeneration of severed axons $(18,46)$. Clinical trials of transplantation of autologous OECs into spinal cord injuries (SCIs) have up to now been based on the more accessible cells obtained from the olfactory mucosa $(6,14,26,27,30,43)$. While these studies serve to establish the safety of the procedure, there was, as in the animal studies, little $(26,27,43)$ or no neurological improvement $(6,30)$. In an experimental study of dorsal root repair in rats, we showed that bulbar, but not mucosal, OECs/ ONFs can make a bridge for severed axons to cross from a peripheral nerve into the spinal cord (18). Based on this, we now describe the results from the treatment of an ASIA A patient (American Spinal Injury Association class A) with an almost total transection of the thoracic spinal cord, who underwent the operation of scar resection, transplantation of cultured autologous bulbar OECs/ ONFs, and reconnection of the spinal cord stumps with autologous sural nerve grafts, followed by a 19-month course of postoperative rehabilitation.

\section{MATERIALS AND METHODS}

\section{Patient}

A 38-year-old male with a complete chronic thoracic SCI was qualified for the study. Thirteen months previously, he had sustained a penetrating SCI at upper vertebral level Th9, caused by a knife assault. Serial magnetic resonance imaging (MRI) studies of the thoracic spine showed an 8-mm-long gap between the spinal cord stumps. The stumps remained connected only by a $2-\mathrm{mm}$ rim of spared tissue in the area of the right spinal cord lateral column (Fig. 1). Initial neurological examination of the patient showed a complete loss of sensory and motor function below the injury (ASIAA), with a zone of partial sensory preservation at level Th9. Transcranial magnetic motor-evoked potentials (MEPs) and electromyography (EMG) excluded any response of the lower extremity (LE) muscles to motor cortex activation and any voluntary muscle activity.

The patient met all the general and neurological criteria to be qualified for the OEC transplantation protocol as described in our recently completed phase I clinical trial (43). However, a diagnosis of chronic allergic sinusitis and nasal polyps (Fig. 2A) was a contraindication for using the olfactory mucosa for obtaining OECs. Initially, the patient was bilaterally anosmic, but after performance of an endoscopic bilateral anterofrontoethmoid sphenoidectomy, he regained his smell perception due to the improved airflow in the nasal cavity (Fig. 2B). In this situation, the only OEC-containing tissue not directly involved in the nasal pathology was the OB. The patient was offered a new two-stage therapeutic approach consisting of the performance of a craniotomy for obtaining
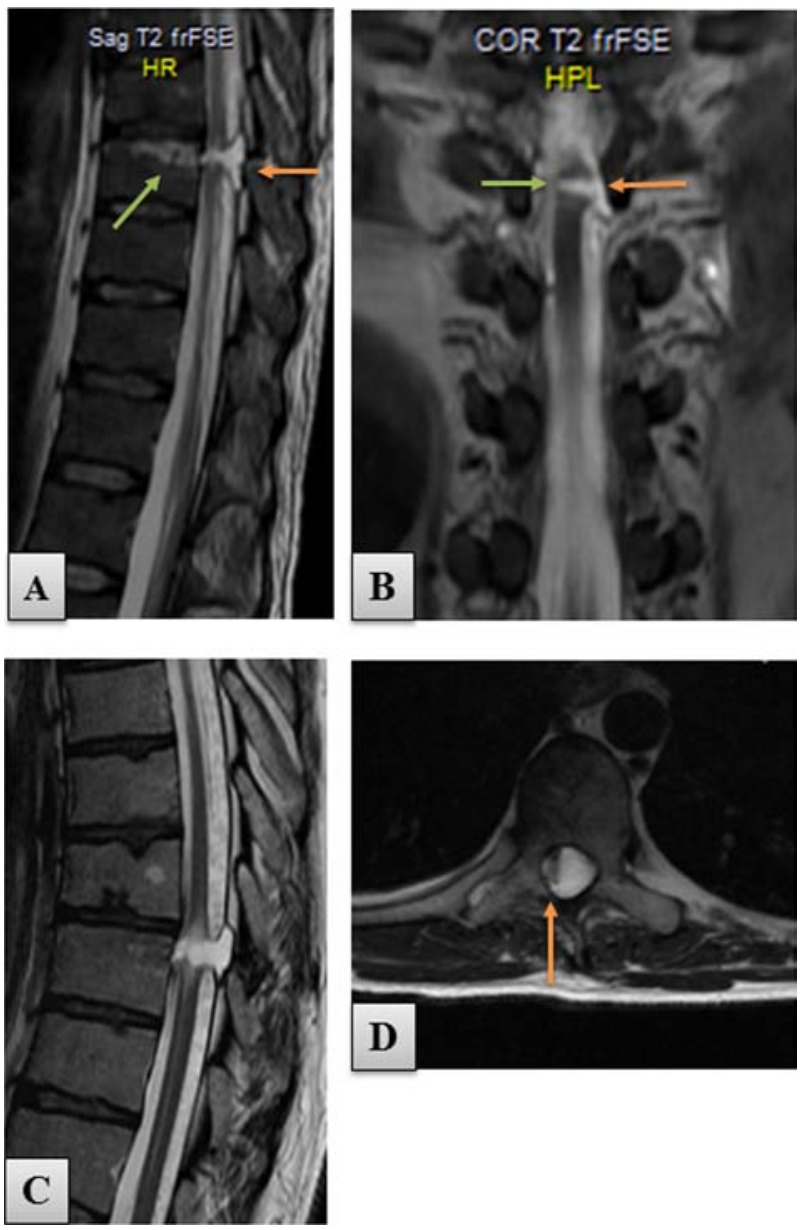

Figure 1. T2-weighted MRI scans of the thoracic spine performed preoperatively. (A) Sagittal view of the spinal cord transection at upper vertebral level Th9. A 5-mm gap of spinal cord continuity (orange arrow) was present 1 month after the injury. Green arrow indicates the area of the vertebral body affected by the knife injury. (B) Coronal scan of the same study showing the area of SCI (orange arrow). Note the thin rim of spared tissue connecting both spinal cord stumps (green arrow). (C) Sagittal MRI scan performed 21 months after the injury. The posttraumatic gap increased its size to $8 \mathrm{~mm}$ because of progressive spinal cord degeneration, seen as hyperdense myelopathic "caps" adjacent to the focus of injury. (D) Axial view of the area of spinal cord transection showing a 2-mm-thick tissue connecting both spinal cord stumps (orange arrow). frFSE, fast relaxation fast spin echo.

one of his OBs for OEC isolation and subsequent transplantation of cultured OECs/ONFs into the lesioned spinal cord. The patient provided a written informed consent according to the Declaration of Helsinki and understood the risk of the trial and the potential for no benefit. The study was approved by the Bioethics Committee of Wroclaw Medical University, according to the guidelines of the National Health Council of Poland. 

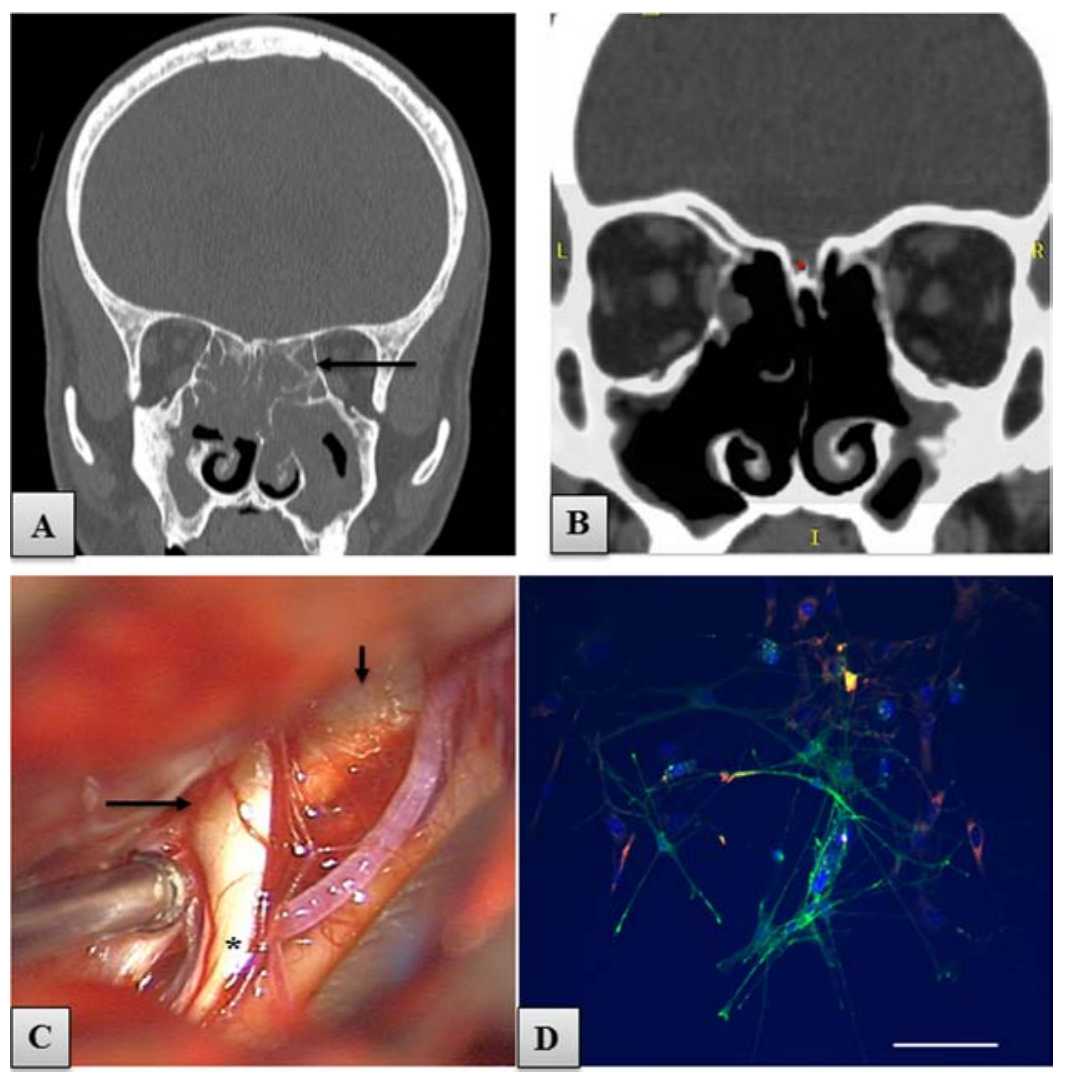

Figure 2. Imaging of head, nasal cavity. and cells to be transplanted. (A) Coronal CT scan of the head. An almost total obliteration of the nasal cavity and paranasal sinuses due to sinusitis is apparent. Black arrow, affected ethmoid sinuses. (B) Coronal CT scan performed after the operation of endoscopic anterofrontoethmoid sphenoidectomy, showing an improved air flow through the sinuses. (C) Photo taken from the operating microscope, showing the region of left olfactory groove with the posterior part of the OB (long arrow), olfactory tract (asterisk), and crista galli (short arrow). (D) Microphotographs of p75 low affinity nerve growth factor receptorpositive (p75/NGFR ${ }^{+}$) bulbar OECs (green) and fibronectin (FN)-positive ONFs (red), taken before cell transplantation, on the 11th day of culture. Hoechst, blue nuclear staining. Scale bar: $100 \mu \mathrm{m}$.

\section{Rehabilitation Protocol}

Before being qualified for the study, it was established that the patient had shown no neurological improvement during a number of different rehabilitation programs over the first 13 months after injury. These programs were incomplete and were interrupted for longer periods because of the necessity for treatment of infections of the respiratory tract, pressure ulcers, and inflammation of the deep venous system of the lower limbs. For this reason, the patient was subjected to an additional intense neurorehabilitation program for 8 months before the planned experimental treatment to confirm that there would be no spontaneous recovery. This program was previously applied in our paraplegic patients qualified for the phase I clinical trial concerning transplantation of autologous olfactory mucosal OECs (43). The patient was trained for $5 \mathrm{~h} /$ day, 5 days/week. The training agenda consisted of $1 \mathrm{~h}$ of range-of-motion and stretching exercises, $3 \mathrm{~h}$ of locomotor training, and $1 \mathrm{~h}$ of sensory training. The main emphasis was set on locomotor training that included training of individual muscle groups of the lower limbs, training for posture and balance, and overground walking exercises. For each exercise, the patient's legs were positioned in a way to have maximal support, and a specific load (using weights attached to ropes) was used to enable the movement in the joints of paralyzed limbs. Each exercise had a fixed number of repetitions. If the patient showed any improvement in the performed exercise, the load was changed in a way to increase the difficulty of the task, as well as increase the number of repetitions. The minimal increase of the load was $100 \mathrm{~g}$. The walking exercise was performed with emphasis to the assessment of the Walking Index (WI) (8), starting from the attempts to stand and walk in parallel bars with braces and the assistance of two persons. Postoperative rehabilitation was planned for at least 24 months. 


\section{Obtaining the Olfactory Bulb}

Before the operation, standard laryngological tests for evaluation of nostril patency and for assessment of respiratory epithelium function (the saccharine test) were performed. Then, the patient's smell perception was tested using a scale for evaluation of smell perception we described previously (43). For obtaining the OB, a left-sided frontolateral craniotomy was performed under general anesthesia. As he had large frontal sinuses, they were opened during craniotomy, cleared from the sinus mucosa, and cranialized at the end of the procedure with autologous muscle and periosteum. Brain relaxation was achieved by a lumbar drainage and by opening of some skull base arachnoid cisterns. Drilling of the skull base ethmoid eminences was necessary because the OB was localized deep inside the olfactory groove. The OB was obtained in two pieces with microsurgical instruments using the subfrontal microscopic endoscopy-assisted approach (Fig. 2C). The choice of the left OB was based on the better smell perception on the left and the lesser atrophy of the left OB in MRI. The tissue samples were maintained at $4^{\circ} \mathrm{C}$ and transported to the culture facility in a complete culture medium consisting of $1: 1, \mathrm{v}: \mathrm{v}$, of Dulbecco's modified Eagle's medium and Ham's F12 (CTS $^{\mathrm{TM}}$ KnockOut $^{\mathrm{TM}}$ DMEM/F12; Gibco, Grand Island, NY, USA), supplemented with $10 \%$ fetal bovine serum (FBS ATMP-Ready, $\gamma$ irradiated; PAA, Pasching, Austria), $1 \mathrm{mM} \mathrm{CTS}^{\mathrm{TM}}$ KnockOut $^{\mathrm{TM}}$ GlutaMAX-I Supplement (complete culture medium, Gibco), $100 \mathrm{U} / \mathrm{ml}$ penicillin, and $100 \mu \mathrm{g} / \mathrm{ml}$ streptomycin (Polfa Tarchomin Warszawa, Poland).

\section{Cell Culture}

Cultures of the patient's bulbar OECs were performed according to Miedzybrodzki et al. (31) with slight modifications. The two fragments of OB tissue were transferred to a $100-\mathrm{mm}$ polystyrene Petri dish (Becton Dickinson, Franklin Lakes, NJ, USA) and washed in $15 \mathrm{ml}$ of CTS ${ }^{\mathrm{TM}}$ DPBS containing calcium and magnesium, without phenol red (Gibco). The blood vessels and meninges were carefully peeled off under a dissecting microscope and the tissue cut into pieces of $2 \mathrm{~mm}$ using a razor blade and incubated at $37^{\circ} \mathrm{C}$ in $0.25 \%$ trypsin solution (Sigma, St. Louis, MO, USA) for $15 \mathrm{~min}$. The enzyme activity was stopped by adding complete culture medium, and the tissue was repeatedly triturated by passing through the tip of a 1-ml pipette (Costar, Corning, Amsterdam, The Netherlands). The suspension was twice spun down at $300 \times g$ for $5 \mathrm{~min}$ and the pellet again gently triturated in $2 \mathrm{ml}$ of complete culture medium. The dissociated cells were seeded on two polystyrene dishes $\left(9.6 \mathrm{~cm}^{2}\right.$, Nunc, Roskilde, Denmark) and one four-well polystyrene plate (1.9 $\mathrm{cm}^{2}$, Nunc) coated with $0.1 \mathrm{mg} / \mathrm{ml}$ poly-L-lysine hydrobromide (PLL, 30-70 kDa, Sigma). The culture dishes were maintained in a humidified incubator at $37^{\circ} \mathrm{C}$ in $5 \% \mathrm{CO}_{2}$. On the fourth day in vitro (DIV), the supernatant containing the nonadherent cells was transferred into new PLL-coated dishes. The cells were fed every second day by replacing half of the complete culture medium volume. On the 12th DIV, the cells became nearly confluent, and the cultures were harvested using CTS ${ }^{\mathrm{TM}}$ TrypLE (Gibco). The enzymatic digestion was stopped by adding complete culture medium, spun down at $300 \times g$ for $5 \mathrm{~min}$, and resuspended. The tubes were placed on ice in a fridge and transported immediately to the operating theater. After five subsequent rounds of washing using $\mathrm{CTS}^{\mathrm{TM}}$ DPBS, the cells were resuspended in appropriate volume of $\mathrm{CTS}^{\mathrm{TM}}$ DPBS and transferred to a glass syringe (World Precision Instruments, Sarasota, FL, USA) for microinjection.

At the 10th DIV, a 5- $\mu$ l aliquot of the cells was fixed with $4 \%$ paraformaldehyde (Sigma) in phosphate-buffered saline (PBS; IITD, Wroclaw, Poland) for $30 \mathrm{~min}$, washed three times in PBS, permeabilized, and blocked with $2 \%$ skim milk (Merck, Darmstadt, Germany) in PBS containing $0.1 \%$ Triton X-100 (Serva, Heidelberg, Germany). Primary antibodies in PBS containing $2 \%$ milk and $0.1 \%$ Triton X-100 were applied overnight at $4{ }^{\circ} \mathrm{C}$. The cells were washed three times in PBS and incubated with fluorescent secondary antibodies in PBS containing 2\% milk and $0.1 \%$ Triton $\mathrm{X}-100$ for $90 \mathrm{~min}$ at room temperature in the dark. After washing four times in PBS, cells were counterstained with Hoechst 33342 (1 $\mu \mathrm{g} / \mathrm{ml}$, Sigma), washed twice with PBS, and mounted using ProLong Gold Antifade (Life Technologies, Rochester, NY, USA). Primary antibodies were 1:100 monoclonal mouse antilow affinity nerve growth factor receptor (anti-p75; clone NGFR5; Invitrogen) and 1:500 polyclonal rabbit anti-human fibronectin Ig (Dako, Glostrup, Denmark). Secondary antibodies were Alexa Fluor 488 goat antimouse $\operatorname{IgG}(\mathrm{H}+\mathrm{L})$ and Alex Fluor 546 goat anti-rabbit IgG $(\mathrm{H}+\mathrm{L})$ (all 1:500; Molecular Probes, Invitrogen, Carlsbad, CA, USA). In all assays, controls were performed by incubating cells with secondary antibodies. Images of fluorescent-labeled cells were captured using a Floid fluorescence microscope (Life Technologies) equipped with Floid Imagine Station Software. Images were exported for further analysis to the ImageJ $1.46 \mathrm{r}$ software (NIH, Bethesda, MD, USA).

\section{Sterility Tests}

A $100-\mu$ aliquot of supernatant from the culture medium was taken on the 5th, 9th, and 12th DIV for the assessment of development of bacterial or fungal infection. The samples were transferred to transport swabs (Hagmed, Rawa Mazowiecka, Poland) and delivered to the Microbiology Department of the Wroclaw Medical University (Wroclaw, Poland). 


\section{Transplantation Procedure}

Preoperative Preparation. The patient was readmitted to the Department of Neurosurgery (Wroclaw Medical University) for the operation of cell transplantation 12 days after $\mathrm{OB}$ retrieval. Based on data from the axial T2-weighted MRI scans, a virtual three-dimensional model of the spinal cord lesion was built. Then a schematic grid for cell microinjection was elaborated with specific topographic reference points on the surface of the spinal cord for intraoperative navigation of the stereotactic injection device, according to our previous experimental protocol (43) (Fig. 3A). Briefly, the cell microinjections were planned to be done in a matrix pattern into the lateral columns of the spinal cord stumps, above and below the lesion epicenter. This matrix consisted of four rows,
$2 \mathrm{~mm}$ apart. The posterolateral sulcus was chosen as the entry point for cell microinjection. The cell suspension was planned to be delivered at five depths at each injection site, $0.5 \mathrm{~mm}$ apart. Another target for cell microinjection was the rim of spared tissue connecting both spinal cord stumps. We also planned to reconstruct the 8-mm gap between the spinal cord stumps with the patient's own peripheral nerve grafts.

Surgical Technique. The patient was placed under general anesthesia in a prone position. After identification of the level of operation with fluoroscopy, a midline skin incision was made, followed by dissection of the paravertebral muscles and laminectomy of the thoracic vertebrae Th7-8-9. Under an operating microscope (OPMI Pentero, Zeiss Company, Jena, Germany), an adhesion between the
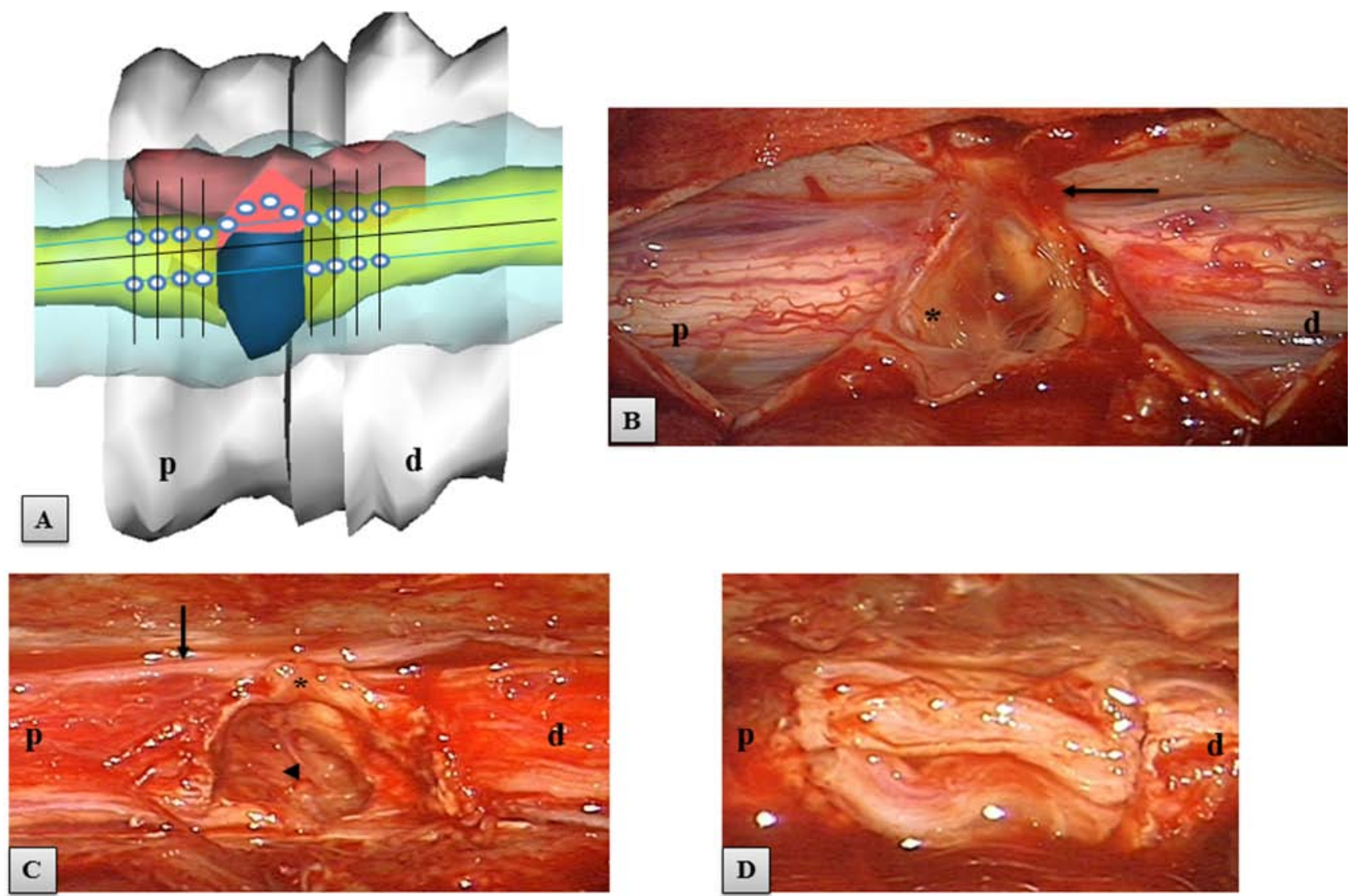

Figure 3. Area of the SCI. (A) A virtual 3D model of the spine and area of SCI. The spinal cord stumps in yellow, the posttraumatic CSF-containing gap in blue, rim of tissue connecting stumps in dark red. For each spinal cord stump, the schematic grid for cell microinjection consisted of four rows (black lines), $2 \mathrm{~mm}$ apart. White dots, the sites of planned cell microinjection in the posterolateral sulcus (blue lines) and in the rim of tissue connecting the stumps; p, proximal spinal cord stump; d, distal stump. (B-D) Intraoperative microscopic images of the area of spinal cord transection. (B) Initial view of the spinal cord after opening of the dura mater. The area of spinal cord transection was covered with yellowish scar tissue (asterisk). Arrow indicates an adhesion between the scar and dura. (C) View of the spinal cord after myeloadhesiolysis and resection of the intraparenchymal scar. This maneuver led to an increase in the initial gap from 8 to $10 \mathrm{~mm}$. Asterisk, the rim of spared tissue connecting both stumps; arrow, a thoracic spinal nerve. Arrowhead, the dura of the ventral surface of the spinal canal, which confirmed that the spinal cord stumps were totally disconnected in this area. (D) View of the spinal cord gap reconstructed with four implanted strips of nerves (two strips were placed ventrally and two strips dorsally), before their fixation with fibrin sealant. 
injured dura and the spinous process Th8 was removed. A midline durotomy was performed, followed by sharp dissection of the posttraumatic adhesions between the spinal cord surface and the dura. The area of SCI consisted of two separated spinal cord stumps, covered with yellowish scar tissue (Fig. 3B). This tissue was removed and specimens taken for histology. This maneuver increased the gap between the stumps from $8 \mathrm{~mm}$ to $10 \mathrm{~mm}$ (Fig. 3C). A 2-mm thin rim of spared tissue connected the margin of the cord stumps on the right. After completion of the myeloadhesiolysis (spinal cord untethering), the system for stereotactic cell microinjection was mounted on the operating table, as described in our previous study (43). Briefly, it was composed of an automatic micropump (UltraMicro Pump III, World Precision Instruments) and a three-dimensional micromanipulator (SM-15, Narishige, Tokyo, Japan). The injector device was fitted with a $25-\mu \mathrm{l}$ glass syringe (World Precision Instruments). The autologous OEC/ONF mixture was suspended in serum-free culture medium, centrifuged, and added to an Eppendorf vial, and a $25-\mu$ l glass syringe with a 26 -gauge beveled needle was filled with the cells. The cell mixture was injected through the posterolateral sulcus between the dorsal nerve rootlets into the lateral columns of the spinal cord, proximally and distally for a distance of $8 \mathrm{~mm}$ on either side of the lesion epicenter. The remaining cell suspension was injected into the rim of tissue connecting both spinal cord stumps. The parameters of cell microinjection are summarized in Table 1 . At the end of the procedure of microinjection, a small aliquot of cell suspension remaining in the Hamilton syringe was taken for sterility tests and monitored in continued cell culture. In the last stage of the operation, a 6-cm-long fragment of the patient's left sural nerve was harvested. Four 12-mm strips of nerve grafts were used for reconnection of the spinal cord stumps (Fig. 3D). The nerves were positioned along the long axis of the spinal cord tracts and were fixed to the spinal cord stumps with fibrin sealant (Tisseel Lyo, Baxter AG, Vienna, Austria). The dura was closed with absorbable sutures. No duraplasty was performed. A wound drain was placed under the muscle layer, and the wound was closed in layers. During the operation, the patient received intravenous methylprednisolone (Solu-Medrol, Pfizer, Kent, UK) in a bolus of $30 \mathrm{mg} / \mathrm{kg}$ (over 15 to
$30 \mathrm{~min}$ ), followed by a constant infusion of $5.4 \mathrm{mg} / \mathrm{kg} / \mathrm{h} \times$ $23 \mathrm{~h}$, together with a prophylactic antacid therapy.

\section{Histopathological Studies}

Immunohistochemical examination was performed on tissue samples taken for routine diagnostic purposes from scar tissue in the spinal cord. Formalin-fixed, paraffinembedded tissue was freshly cut $(4 \mu \mathrm{m})$. Immunohistochemistry was performed as previously described (17) using the following antibodies diluted in Antibody Diluent, Background Reducing (Dako): epithelial membrane antigen (EMA; clone E29; monoclonal mouse, dilution 1:100, Dako), S100 (rabbit polyclonal, dilution 1:400, Dako), neurofilament (NF; clone 2F11; monoclonal mouse, dilution 1:100, Dako), vimentin (clone V9; monoclonal mouse, dilution 1:100, Dako), and glial fibrillary acidic protein (GFAP; rabbit polyclonal, dilution 1:500, Dako). Hematoxylin-eosin (Sigma) counterstaining was performed.

\section{Initial and Repeated Assessments}

The patient's medical condition was evaluated regularly pre- and postoperatively. This included general medical assessment, otorhinolaryngological, neurological, physiotherapeutic, and psychological tests, as well as radiological and neurophysiological studies. All studies were performed by the same assessors. They were not blinded.

A detailed general medical assessment was conducted preoperatively and in the first 5 weeks postsurgery and included hematology, blood chemistry, and urine analysis. Tests for the presence of anti-HIV antibodies were performed preoperatively, and hepatitis B and C status was assessed. Standard electrocardiographic studies and chest X-rays (Philips, Amsterdam, Netherlands) were also performed. Microbiological studies of blood, urine, or cerebrospinal fluid (CSF) were planned to be conducted only in case of suspicion of infection.

Laryngological examination included smell perception tests, as well as evaluation of computed tomography (CT) and MRI scans of the head, nasal cavity, and paranasal sinuses.

Neurological examination was performed monthly and included ASIA examination, examination of deep

Table 1. Parameters of Cell Microinjection

\begin{tabular}{|c|c|c|c|c|c|c|}
\hline $\begin{array}{l}\text { Volume of } \\
\text { Single Injection }\end{array}$ & $\begin{array}{l}\text { Velocity of } \\
\text { Cell Injection }\end{array}$ & $\begin{array}{l}\text { Total Number } \\
\text { of Injections }\end{array}$ & $\begin{array}{c}\text { Number of } \\
\text { Injection Sites }\end{array}$ & $\begin{array}{c}\text { Total } \\
\text { Volume }\end{array}$ & $\begin{array}{c}\text { Total Number } \\
\text { of Grafted Cells }\end{array}$ & $\begin{array}{l}\text { Percentage of } \\
\text { OECs in the } \\
\text { Cell Suspension }\end{array}$ \\
\hline$\overline{0.5 \mu \mathrm{l}}$ & $2 \mu \mathrm{l} / \mathrm{min}$ & 96 & 24 & $48 \mu \mathrm{l}$ & 500,000 & $16 \%$ \\
\hline
\end{tabular}

OECs, olfactory ensheathing cells. 
sensation, evaluation of spasticity using the Ashworth scale, and assessment of reflex activity and the Medical Research Council (MRC) muscle scale.

A spinal injuries physiotherapist undertook regularly a Functional Independence Measure (FIM), evaluated the WI, and recorded the achievements in each physical exercise during the locomotor training.

Psychological evaluation was performed preoperatively and at 1,12 , and 19 months after surgery. A clinical interview as well as the following psychological tests were conducted: the Minnesota Multiphasic Personality Inventory-2 test (MMPI-2) (2), the Generalized SelfEfficacy Scale (GSES) (19), the State-Trait Anxiety Inventory test (STAI) (42), and the Eysenck Personality Questionnaire-Revised (EPQ-R) (10). A cultural adaptation of these tests was routinely applied.

MRI images were undertaken on a 1.5-Tesla MR unit (GE Signa HDx, Milwaukee, WI, USA) and included T2-weighted images, T2-weighted fat saturation (FATSAT) images, and T1-weighted images before and after administration of contrast medium (gadolinium; Multihance, Bracco Diagnostics, Singen, Germany) in the sagittal, coronal, and axial planes. Diffusion tensor imaging (DTI) included an assessment of the topography of the water diffusion tracts in the spinal cord on tractography and the estimation of the values of fractional anisotropy (FA) of the spinal cord on the FA maps, at 0.5 and $2 \mathrm{~cm}$ above and below the lesion epicenter. MRI and DTI studies were performed preoperatively and at 1, 5, 8, 12, and 17 months postsurgery according to the previously described protocol (43).

Neurophysiological examinations included transcranial magnetic MEPs, electroneurography (ENG), and EMG using the KeyPoint Diagnostic System (Medtronic, Copenhagen, Denmark), as previously described (43). Neurophysiological testing was performed twice before surgery and at 1, 5, 8, 11, 14, and 17 months postoperatively. During the MEP study, three positive recordings with similar amplitudes and latencies of potentials were recorded from rectus abdominis, rectus femoris, and extensor digiti muscles on both sides to show the integrity of long efferent neural transmission. Beside wellknown parameters of amplitudes and latencies in MEP recordings, the duration of potentials measured from the onset to the end with the reference to isoelectric line were also recorded. Bilateral EMG recordings from the rectus abdominis, rectus femoris, gastrocnemius, anterior tibial, and extensor digiti muscles were assessed using surface electrodes, and needle electrodes were used to increase the measurement precision when the patient was asked to perform the voluntary contractions lasting $5 \mathrm{~s}$.

The urodynamic study was conducted according to the protocol of the International Continence Society (44) using the Duet Logic G2 system (Medtronic). It consisted of uroflowmetry, pressure flow studies, and EMG of the anal sphincter. It was performed before surgery and at 1, 5 , and 12 months postoperatively.

\section{Statistical Analysis}

Statistical analyses were performed using Statistica 10 (StatSoft, Inc., Tulsa, OK, USA) A value of $p=0.05$ was considered significant. The Mann-Whitney $U$ test was applied for assessment of statistical differences in measured variables. The Spearman Rank test was used to test correlations between specific measurements and features. Statistically significant correlation coefficients greater than 0.50 were considered important.

\section{RESULTS}

Recovery After the Operation of Olfactory Bulb Retrieval

The operation for obtaining the OB via craniotomy was safe. No neurological or general complications were noted postoperatively. The head CT scan performed 2 days after the surgery did not show any abnormalities. Shortly after the operation, the patient lost his smell perception on the left, where the bulbectomy was performed, but unexpectedly, follow-up showed a later, partial recovery of olfaction on the bulbectomized side. This persisted for the period of 19 months of observation (Fig. 4).

\section{Cell Culture}

The cultures of cells isolated from the OB were not purified and contained mainly ONFs and OECs and comprised more than $95 \%$ of the Hoechst-stained cell population. OECs were identified as bi- or multipolar p75-NGFR-positive cells, with small cytoplasm and thin, long processes. They formed a network on a monolayer of flattened FN-positive ONFs (Fig. 2D). The percentage of p75-NGFR-positive cells was $16 \%$. After 12 days, when OECs reached a confluent monolayer in culture, they were detached from the culture flasks and prepared for transplantation. The sterility tests of the cell culture, performed every 5 days, showed no evidence of bacterial or fungal contamination within the whole period of cell culture. The residual volume of cells remaining in the Hamilton syringe at the end of the operation of cell microinjection was seeded onto culture flasks and cultured for about 10 days for identification of cell populations and for assessment of culture sterility. In all cultures used for transplantation, p75-NGFR-positive cells could be identified in culture, and there was no evidence of microbial contamination.

Assessment of the Safety of the Operation of Cell Transplantation and Spinal Cord Reconstruction

The operation of myeloadhesiolysis and glial scar resection followed by cell microinjection and bridging of 


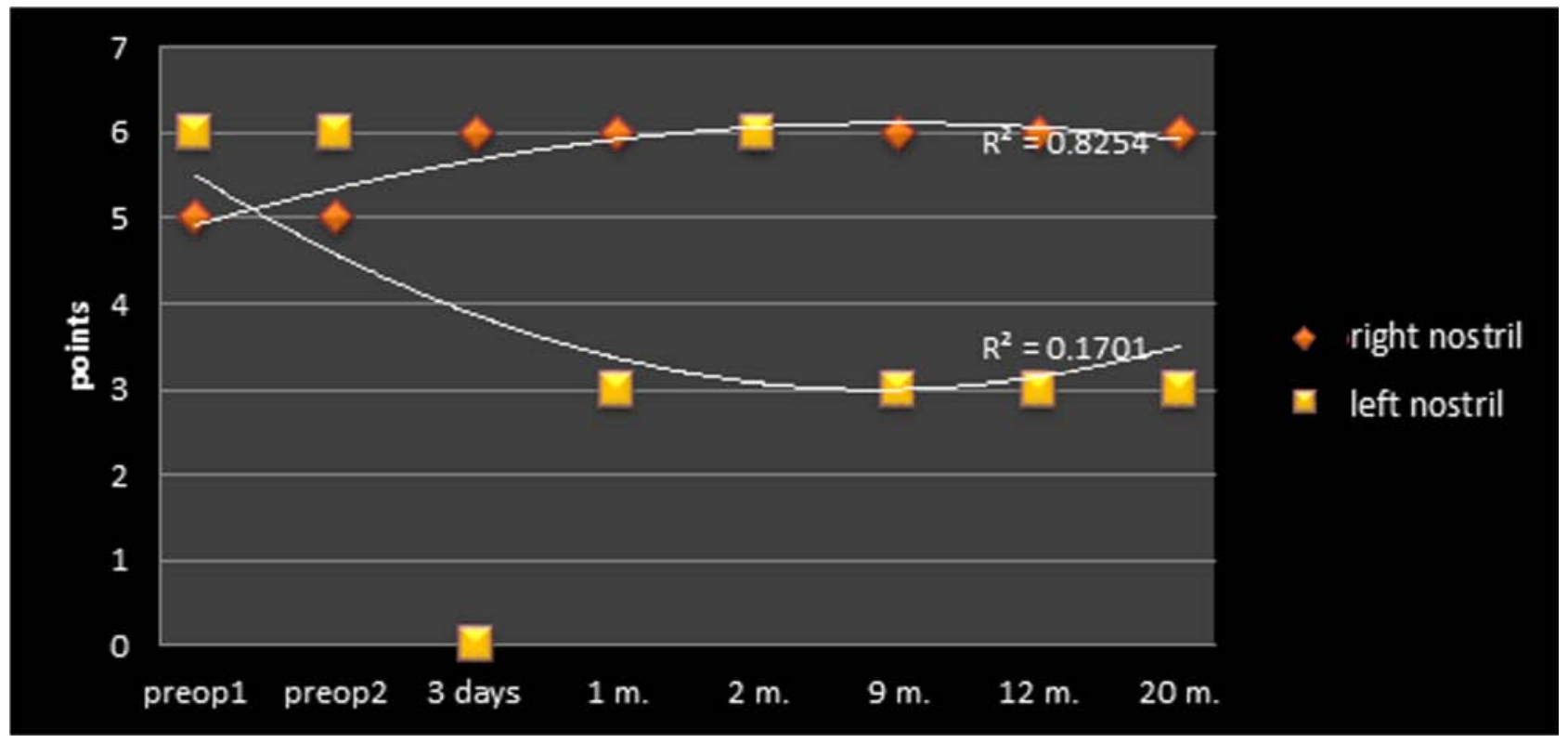

Figure 4. Summary of the smell perception test performed for the right and left nostril pre- and postoperatively. The maximal achievement that can be assigned for each tested side was six points. Note that after removing the left OB, the patient did not totally lose olfaction on the left but remained hyposmic.

the spinal cord with autologous peripheral nerve grafts was safe over the period of 19 months of observation. We did not observe neurological deterioration, neuropathic pain, clinical or laboratory evidence of neuroinfection, nor any general medical complications attributed to the surgical intervention. The patient spent the first 2 days after the surgery in the intensive care unit to have optimal control of his medical condition. In the first 3 days after the surgery, he was suffering mainly from pain from the operative wound, requiring treatment for a period of several days with morphine (Morphini Sulfas, Polfa, Warszawa, Poland) administered subcutaneously at a dose of $5 \mathrm{mg}$ every $6 \mathrm{~h}$. There was no spine instability or stenosis, as well as no evidence of myelomalacia, edema, inflammation, or tumors of the spinal cord at the injection site, as documented in the five postoperative MRI studies (Fig. 5). MRI showed a good integration of the implanted nerve strips with the host spinal cord tissue. The nerve implants were well vascularized (Fig. 5B) and retained their size (Fig. 5G). T2-weighted scans showed some mild degenerative changes in the spinal cord stumps that were not progressive and were not associated with any negative influence on the clinical state of the patient and/ or the results of his electrophysiological studies (Fig. 5).

The preoperative DTI study revealed that a gap in continuity of diffusion tracts in the spinal cord was at the level of the SCI (Fig. 6). An early postoperative DTI study, performed at 5 weeks, showed restitution of the tracts of water diffusion across the area of implanted nerve grafts and also across the rim of spared tissue connecting the spinal cord stumps. Yet tractography studies at 17 months after operation again showed a gap in the continuity of diffusion tracts, which did not correlate with the continued neurological and neurophysiological recovery of the patient (Fig. 6). Assessment of the values of FA did not show significant changes between pre- and postoperative studies (Table 2).

\section{Histopathological Findings}

Histopathological examination of the specimen obtained from the resected intraparenchymal scar tissue showed no astroglial reactivity but fibrous connective tissue intermingled with bundles of peripheral nerve fibers and Schwann cells (Fig. 7).

\section{Neurological Assessment}

Preoperatively, the patient presented, in serial examinations, symptoms of a complete SCI (ASIA A), with a zone of partial preservation (ZPP) at dermatomal level Th9 and complete loss of any type of sensation below this dermatome, including the S4-S5 dermatomes. The patient was assigned 31 points for each side in the ASIA Light Touch and Pin Prick Score. There was a paralysis of the leg muscles ( 0 points according to the MRC scale) and weakness of the lower trunk muscles causing trunk instability (positive Beevor's sign) during the attempts of the patient to stand. Increased spasticity was present in the LEs between 4 and 5 on the Ashworth scale, with a bilaterally positive Babinski sign and LE hyper-reflexia, except in the ankles, where there was no Achilles tendon 

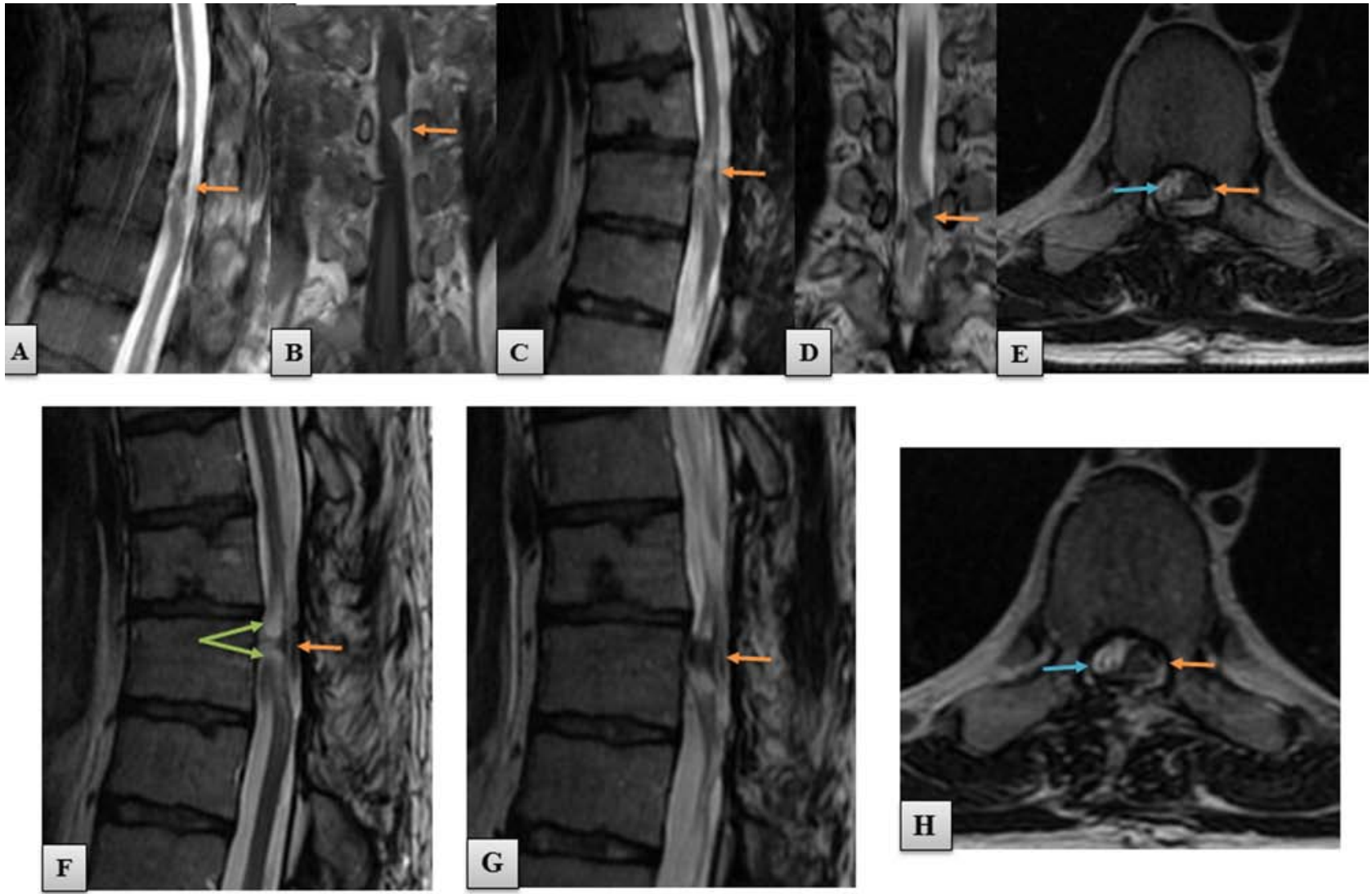

Figure 5. MRI studies of the spinal cord at set time points after treatment. MRI studies performed at 5 weeks (A,B), 5 months (C,D,E), and 17 months $(\mathrm{F}-\mathrm{H})$ after the spinal cord operation. Orange arrows, implanted sural nerve strips. (A) On the sagittal T2-weighted image, the nerve implant appeared as a hypodense structure that reconnected effectively the sectioned spinal cord stumps. (B) Coronal scan of the same area showing that after contrast administration the nerves were hyperdense, which confirms their good vascularization. Implant size was $1.3 \times 0.7 \mathrm{~cm}$. T2-weighted sagittal (C) and coronal scans (D) after 5 months confirmed the integrity of the nerve grafts with the host tissue. (E) Axial T2-weighted scan shows that the nerve grafts (orange arrow) filled most of the surface connecting the spinal cord stumps, while the rim of spared tissue was hardly visible (blue arrow), when compared with preoperative MRI scans (Fig. 1D). (F) Sagittal T2-weighted image at 17 months showed a hyperdense area of mild spinal cord degeneration that was present in both pre- as well as all postoperative studies (green arrows). (G) The sural nerve autografts retained their size when compared with the early MRI study. (H) At 17 months postsurgery, the nerve grafts still were the dominant structure found in the area of spinal cord reconstruction (orange arrow). The spared tissue was also identifiable (blue arrow).

reflex. Additionally, the legs were constantly cold due to an impaired vascular autoregulation. A small pressure ulcer was present on the lateral surface of the right hip, which was not painful because of the sensory impairment.

After the operation, the patient's neurological state did not change significantly during the first 4 months. Five months after surgery, the first signs of recovery of sensation in dermatomes S4-S5 were present, becoming more evident at 6 months. A gradual recovery of superficial sensation, particularly in the right LE, was observed during the whole postsurgical period of 19 months, increasing in pace after the 10th month. The ASIA Light Touch score reached 35 points for the right leg, while it was 32 in the left leg. The increase in the light touch score for the period after 10 months postsurgery was statistically significant for both LEs $(p<0.05)$ (Fig. 8). The patient also achieved a higher score in the ASIA Pin Prick Test (42 for the right leg and 34 for the left) but was statistically significant only for the right leg $(p<0.05)$ (Fig. 8). For the first time, the patient reported pain evoked by irritation of the small pressure ulcer present on the right hip. We also noticed a recovery of the deep sensation in the legs. At 6 months postsurgery, the patient began to feel the tension applied to his leg muscles during training and the movement of his joints. Between the 10th and 19th months after surgery, the recovery of deep sensation was confirmed with specific tests such as the vibration test or tests for evaluation of limb position. On about 30 trials, the patient could determine, with $75-85 \%$ accuracy, the direction of movement of his feet with his eyes closed and even could discriminate the movement of his toes from the movement of the whole foot. 

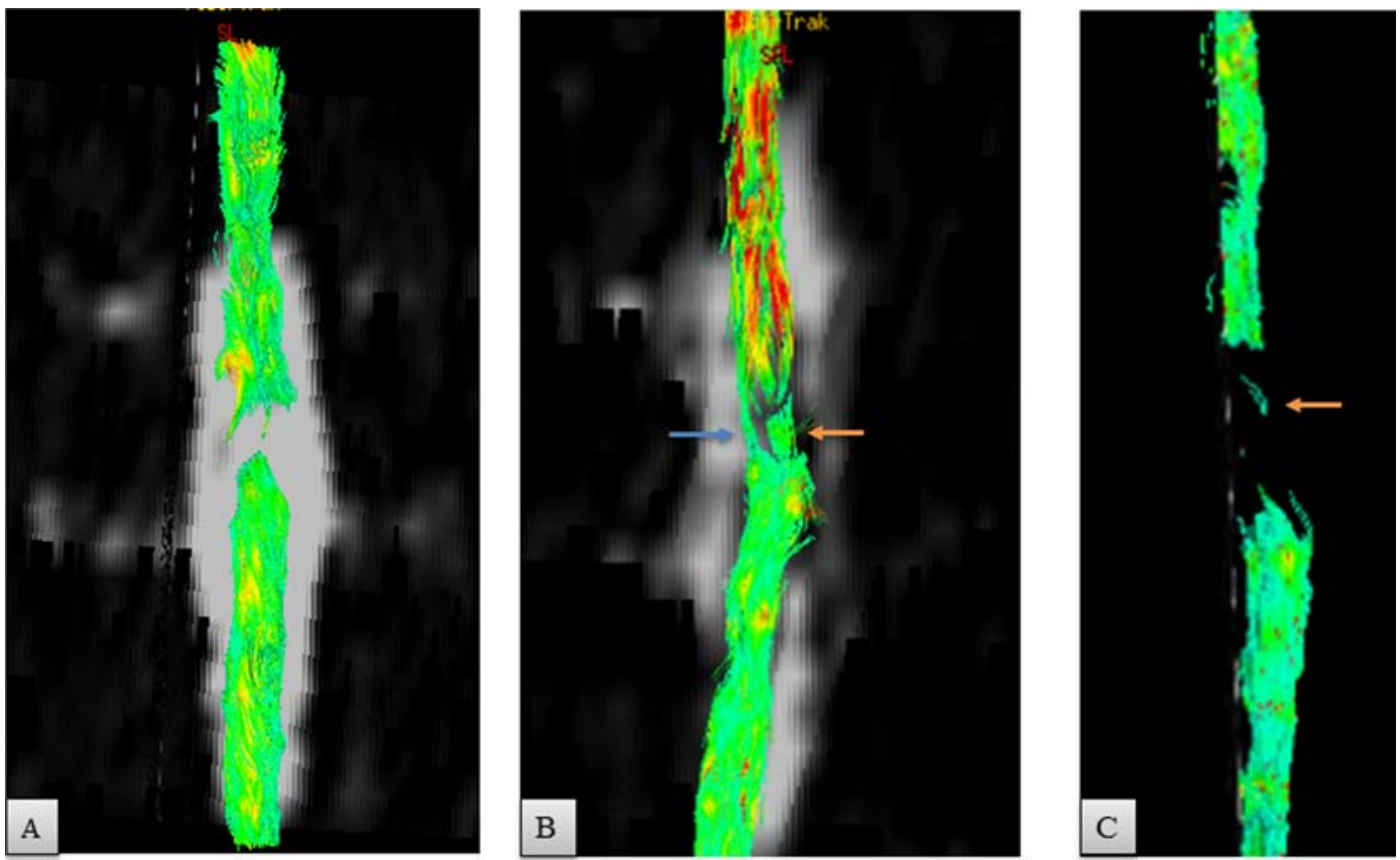

Figure 6. Scans of spinal cord tractography. (A) An 8-mm gap of continuity of diffusion tracts was observed preoperatively. (B) Five weeks after the operation, the tracts of water diffusion crossed through the area of implanted nerves (orange arrow) and the rim of spared tissue connecting the stumps (blue arrow). (C) At 17 months, a gap of $1.8 \mathrm{~cm}$ was present. Orange arrow points out either thin tracts at the level of the implant or an artifact.

Simultaneously with the improvement of superficial and deep sensation, we observed an evident recovery of voluntary motor function in the previously paralyzed muscle groups. This recovery appeared in a segmental pattern, starting from the lower part of the abdominal and other trunk muscles. This was first evident 5 months after surgery. The recovery of voluntary function of selected LE muscles was preceded by a marked increase in muscle mass of the left thigh 4 months postoperatively, causing visible leg asymmetry. The circumference of the left thigh, measured in the middle of the line connecting the anterior superior iliac spine and the patella, was $50 \mathrm{~cm}$ versus

Table 2. Summary of the Values of Fractional Anisotropy

\begin{tabular}{lcccc}
\hline Date & $+2 \mathrm{~cm}$ & $+0.5 \mathrm{~cm}$ & $-0.5 \mathrm{~cm}$ & $-2 \mathrm{~cm}$ \\
\hline Preop. & 0.305 & 0.266 & 0.366 & 0.415 \\
Preop. & 0.397 & 0.399 & 0.424 & 0.468 \\
1 month & 0.480 & 0.323 & 0.327 & 0.545 \\
5 months & 0.407 & 0.287 & 0.504 & 0.487 \\
8 months & 0.316 & 0.377 & 0.436 & 0.574 \\
17 months & 0.363 & 0.280 & 0.419 & 0.476 \\
\hline
\end{tabular}

FA values were measured in the spinal cord above and below the lesion epicenter. Levels $0.5 \mathrm{~cm}$ and $2 \mathrm{~cm}$ refer to the proximal spinal cord stump, whereas levels $-0.5 \mathrm{~cm}$ and $-2 \mathrm{~cm}$ to the distal stump. Preop., preoperatively.
$42 \mathrm{~cm}$ in the right thigh and increased gradually from 50 to $54 \mathrm{~cm}$ within a period of 18 months. The increase in muscle mass in the right thigh did not exceed $2 \mathrm{~cm}$ in the same period. There was no change in the mass of the calf muscles. As the patient had sustained inflammation and thrombosis of the LE deep venous system in the preoperative period, Doppler ultrasonography (Vivid 7, GE Healthcare, Horten, Norway) of the veins and arteries of the LE was performed. The independent angiologic study excluded venostatic edema or postthrombotic syndrome as a cause of the increased circumference of the left leg.

The motor recovery was more prominent in the left LE. The first voluntary adduction of the left leg was observed 5 months after surgery and increased with time, reaching level 3 according to the MRC scale at 11 months. Ten months postoperatively, a voluntary adduction of the right leg (MRC 2) was noted and slight hip flexion on the left (MRC 1). The increase in the strength of the left and right adductor muscles was statistically significant for the period after 7 months postsurgery $(p<0.05)$ (Fig. 9). The first voluntary knee extension was recorded on the left (MRC 2) at 1 year and was confirmed in later studies. A slight knee extension in the right leg was also noted at 14 months postsurgery (MRC 1). As a result, the patient's neurological state turned 6 months after surgery from ASIA A to ASIA B and at 11 months to ASIA C 


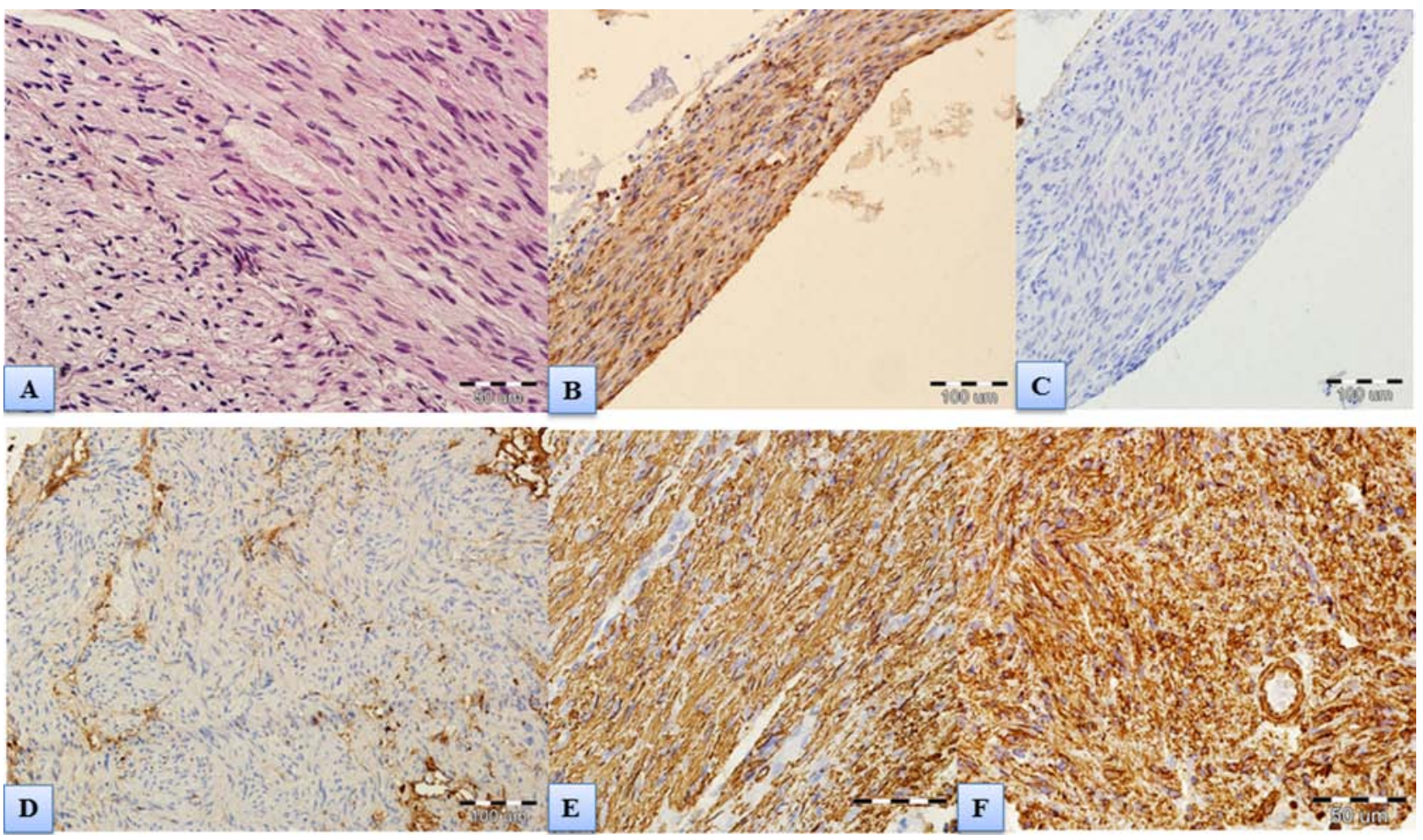

Figure 7. Spinal cord scar. (A) Scar composed of peripheral nerve fibers and fibrous connective tissue without CNS tissue; hematoxylin and eosin staining. (B) Immunohistochemical expression of S100 protein confirming aligned clusters of Schwann cells with typical ovoid nuclei within the scar. (C) Lack of immunohistochemical expression of EMA excluding presence of meningeal tissue. (D) Negative immunohistochemical staining for glial fibrillary acidic protein (GFAP) proving no glial components. (E) Immunohistochemical expression of NF typical for nerve fibers (scale bar: $50 \mu \mathrm{m}$ ). We assumed that these are the central branches of dorsal rootlets lying in the scarred area. (F) Immunohistochemical expression of vimentin corresponding with connective tissue.

(Fig. 10). The observed increase of ASIA motor scores for the left and right LEs was statistically significant for the period after 10 months when compared with the preoperative state and the first 10 months after surgery $(p<0.05)$ (Fig. 9).

A decrease of spasticity in both LEs was noted postoperatively but was significant statistically only for the right $(p<0.05)$. The mean Ashworth score decreased from 5 to 3.8 for the left LE and from 4 to 3 for the right. At 5 months postoperatively, the Babinski sign disappeared bilaterally, and the Achilles tendon reflex reappeared in the left LE. The left LE seemed also to have better vascular autoregulation when compared to the right.

\section{Physiotherapeutic Assessment}

During the period of 8 months of intense preoperative rehabilitation, the patient did not show any improvement in performed physical exercises. There was asymmetrically increased spasticity of the trunk and LE, paralysis and loss of sensation of the LE, and the equinovarus positioning of the left foot. This hindered any attempts of patient tilting and made walking in braces in parallel bars impossible. WI was 0 .

Increased strength of the trunk muscles, the first voluntary movements of adduction and abduction of the left leg, decrease in muscle spasticity, and the evident recovery of proprioception in the LE, which started to be visible from the sixth month after surgery, increased the quality of performed exercises and enabled the introduction of more difficult tasks. The increase of the muscle strength and coordination enabled better trunk, pelvis, and hip stabilization and could prepare the patient for the first exercises of walking reeducation. In the period from 9 to 11 months after surgery, there was an evident improvement in the technique of exercise performance and an increase in the values of the loads in exercises requiring a high degree of voluntary function of abdominal and back muscles, gluteal muscles, adductors and abductors, hip flexors, and knee extensors. The left leg was dominant in muscle mass, number of voluntary controlled muscles, and time of appearance of first symptoms of motor recovery. This enabled the introduction of separate exercises for the left 

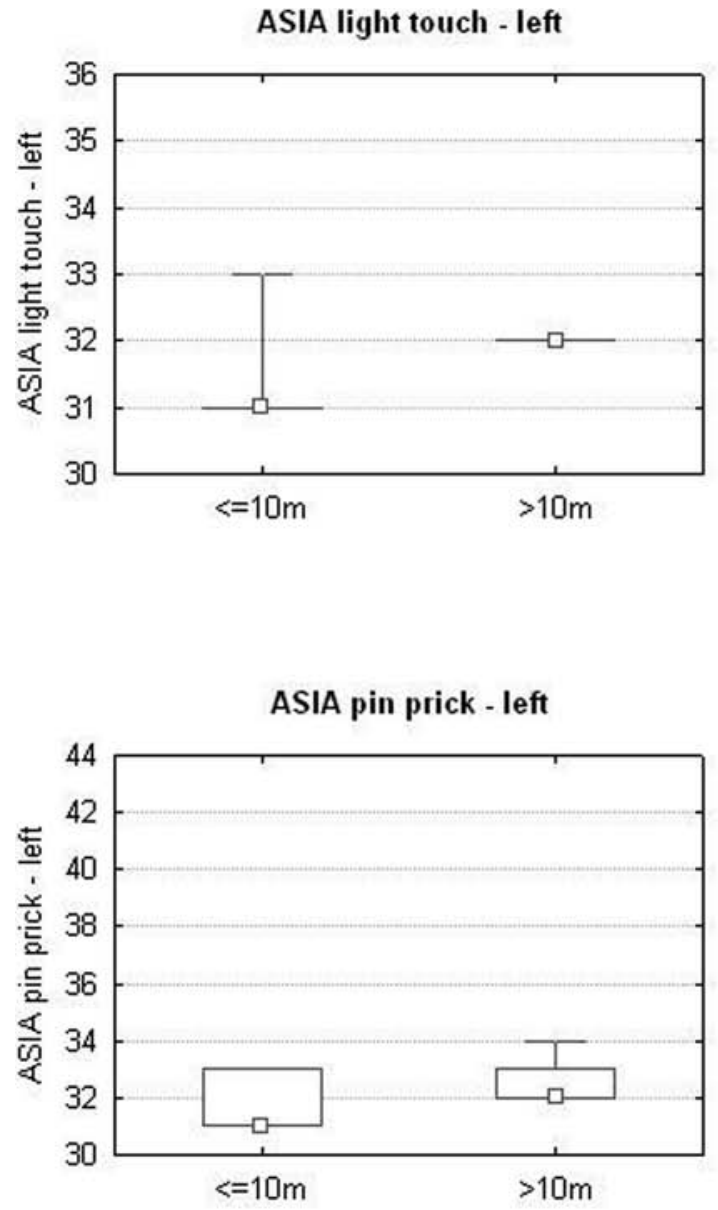
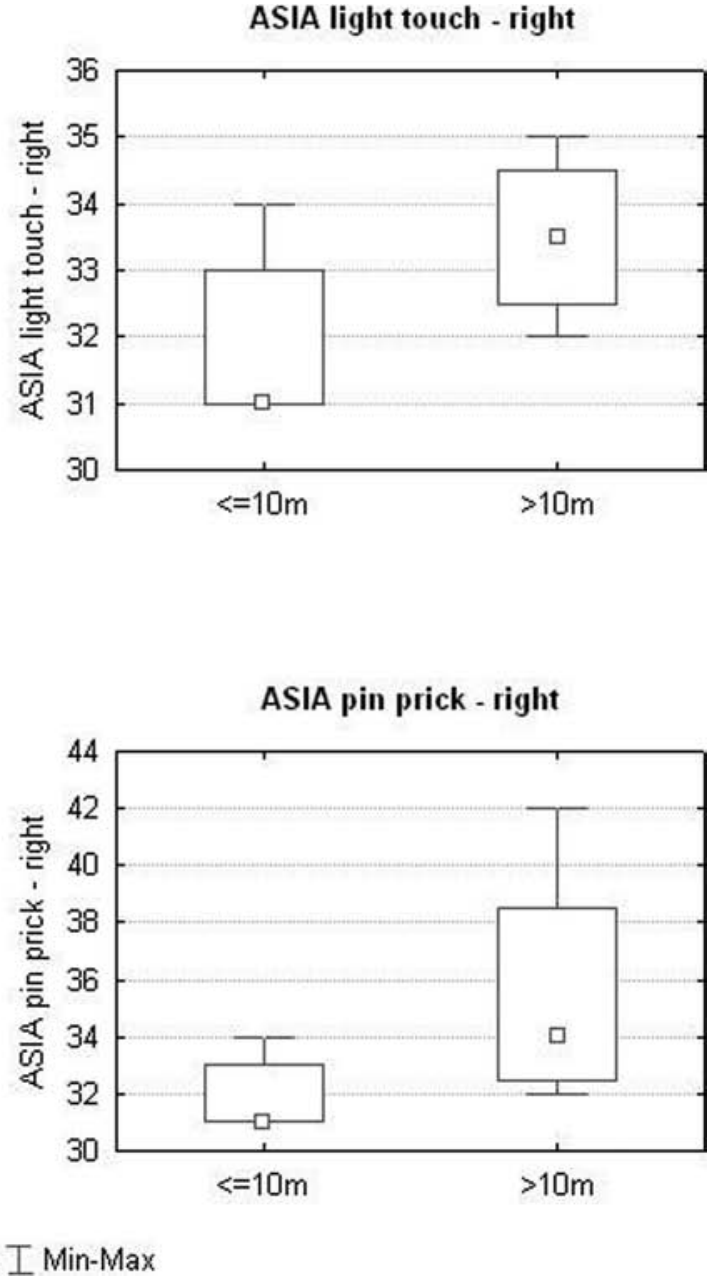

Figure 8. Summary of the results from the light touch and pin prick tests. The observed improvement in these tests in the period after the 10th postoperative month was statistically significant $(p<0.05)$ when compared to the preoperative state and the first 10 months postsurgery. There was also more evident recovery of superficial sensation in the right LE when compared with the left. ASIA, American Spinal Injury Association.

and right leg. We did not observe any notable improvement in the exercises testing muscles of the posterior part of the thigh and the calf. Table 3 summarizes the results from physiotherapy.

The observed improvement in different static physical exercises led gradually to the first attempts to walk. Six months postsurgery, the patient was able to ambulate $10 \mathrm{~m}$ in parallel bars with long leg braces and the physical assistance of one person (WI $=3$ ); at 13 months, WI increased to 5 (parallel bars, no physical assistance), and starting from 14 months, the patient was able for the first time to ambulate with a walker, long braces, and the assistance of one person (WI=6). Additionally, in the last months of observation, the patient started to walk both in parallel bars and with a walker with short braces, locked only at the ankles. The observed increases in the WI values after surgery were statistically significant $(p<0.05)$.
Measurements of functional activity using the FIM scale showed a significant increase in the FIM values from 102 to 104 points before surgery to $116-120$ points in the period between the 6th and 11th months, to reach 123-124 points between the 12th and 19th months.

\section{Correlations Between the Results From Neurological and Physiotherapeutic Studies (Table 4)}

A statistically significant strong positive correlation $(p<0.05)$ was found between the ASIA motor scores for both LEs, the scores obtained for leg adduction, and the light touch and pin prick scores in the right and left LE; between the left and right ASIA motor scores and the WI and the right light touch and pin prick scores and WI; and between the WI and the FIM score. A negative correlation was noted between the leg adduction tests and the leg spasticity measured with the Ashworth score. 

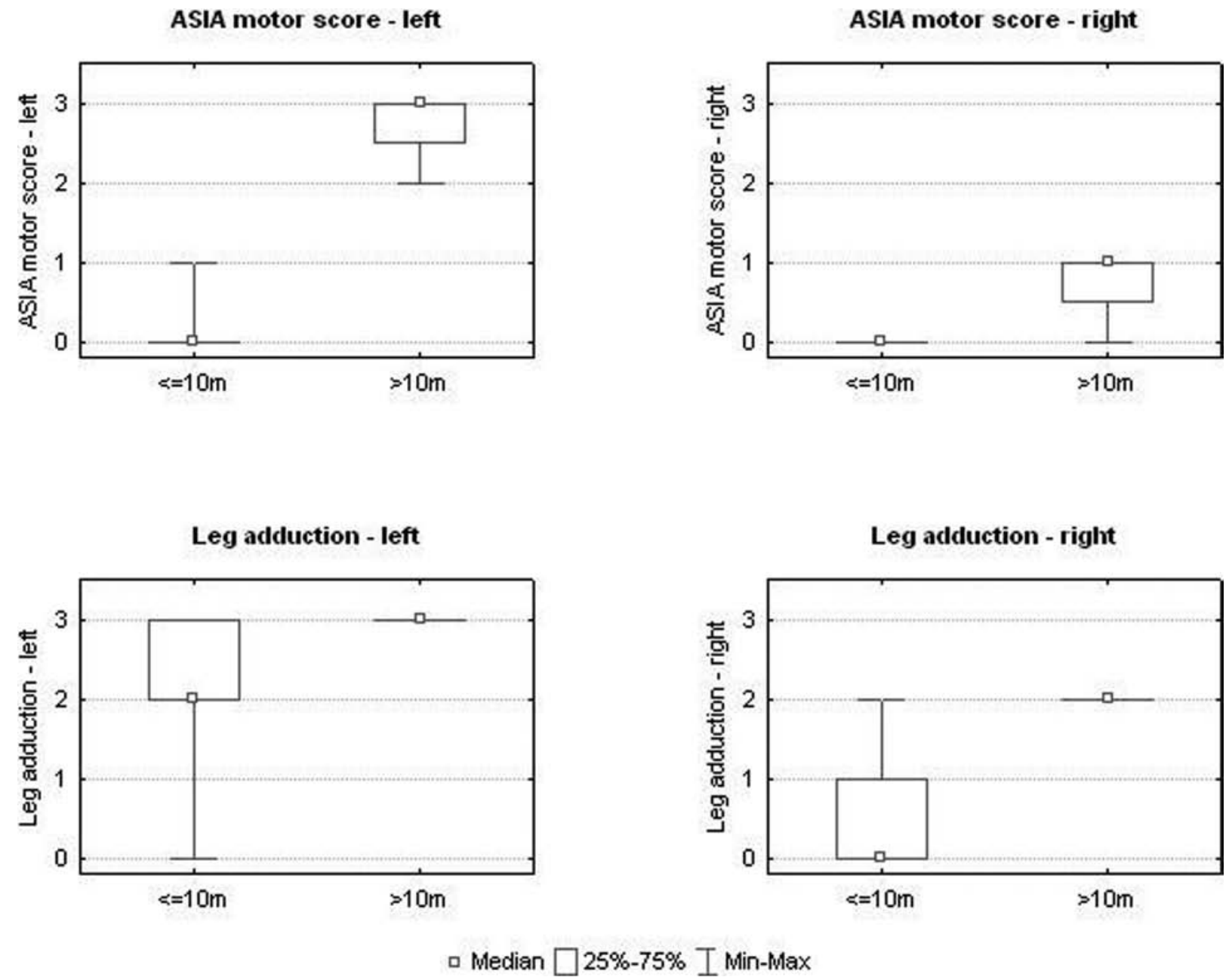

Figure 9. Graphs showing the results from motor tests of the LE. A voluntary muscle activity could be observed in both the left and right LE but was more evident after the 10th postoperative month and achieved higher MRC scores in the left one.

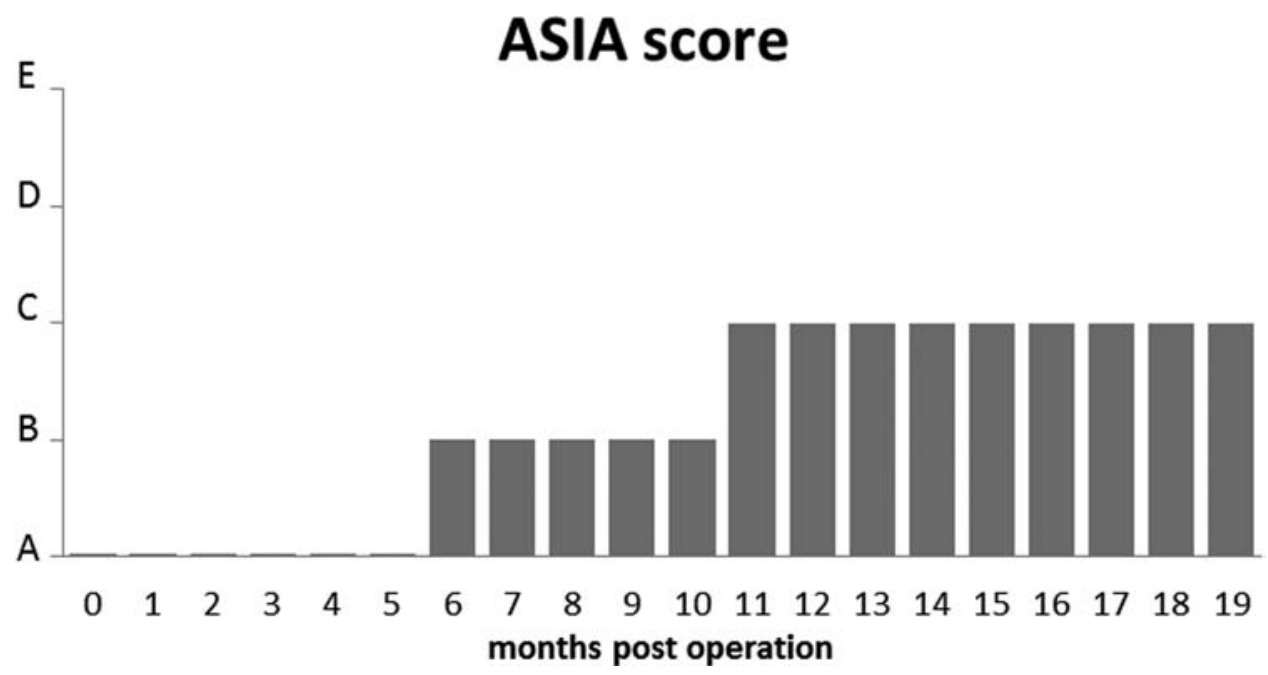

Figure 10. Chart showing the changes in the ASIA score in the postoperative period. ASIA Score increased to B around 6 months and $\mathrm{C}$ around 11 months. 
Table 3. Summary of the Achievement From Selected Rehabilitation Exercises

\begin{tabular}{|c|c|c|c|c|c|c|c|c|}
\hline No. & $\begin{array}{c}\text { VII-XII } \\
2011\end{array}$ & $\begin{array}{l}\text { I-III } \\
2012\end{array}$ & $\begin{array}{l}\mathrm{O} \\
\mathrm{p}\end{array}$ & $\begin{array}{l}\text { V-VII } \\
2012\end{array}$ & $\begin{array}{c}\text { VIII-XII } \\
2012\end{array}$ & $\begin{array}{l}\text { I-III } \\
2013\end{array}$ & $\begin{array}{c}\text { V-VIII } \\
2013\end{array}$ & $\begin{array}{c}\text { IX-XII } \\
2013\end{array}$ \\
\hline 1 & $-8,750$ & $-8,000$ & e & $-1,000 /-1,000$ & $-1,000 /-1,000$ & w.h. & w.h. & w.h. \\
\hline 2 & 10,350 & 8,100 & $\mathrm{r}$ & 7,050 & 8,300 & - & 7,500 & 9,050 \\
\hline 3 & - & - & a & - & - & 4,200 & 3,990 & 3,900 \\
\hline 4 & - & - & t & - & - & 4,200 & 4,750 & 5,100 \\
\hline 5 & - & - & 1 & - & - & - & 0 & 1,000 \\
\hline 6 & - & - & o & - & - & - & $2,500-5,000$ & 8,750 \\
\hline 7 & - & - & $\mathrm{n}$ & - & - & $-3,000$ & $-3,000$ & $-3,000$ \\
\hline 8 & - & - & & - & $-11,450$ & $-5,500$ & w.h. & w.h. \\
\hline 9 & $-3,000$ & $-3,250$ & & $-2,700$ & $-3,600$ & $-4,200$ & $-4,200$ & $-4,100$ \\
\hline
\end{tabular}

Roman numerals refer to the month. Positive numbers show the value of applied load (in grams). Negative numbers show the value of load relief. Minus sign indicates that the patient was unable to perform the exercise. 1. Forward bends from a supine position—a test of abdominal muscle strength. In this case, the patient was initially holding a bar connected with a suspended load and started the exercise from an angle of $30^{\circ}$. Starting from 9 months after surgery, he could bend forward without any help (w.h.), beginning the movement from a completely supine position $\left(0^{\circ}\right)$. 2. Hip extension—an exercise testing trunk muscles and gluteal muscles and performed in a supine position with suspended straight legs. Preoperatively, the patient was able only to initiate a simultaneous downward movement of both legs when blocked in long braces. This leg movement was triggered by the use of trunk musculature, but further movement was prevented by increased spasticity and muscle paralysis. After surgery, he was able to perform a full range of hip extension without leg immobilization in braces. In addition, the patient became able to perform the same exercise with his legs suspended separately (No. 3, right leg; No. 4, left leg). The left leg was able to overcome much higher loads than the right. 5. Leg abduction in a sitting position. 6. Leg adduction in a sitting position. 7. Drawing of suspended knees toward the abdomen using hip flexors (iliopsoas muscles and rectus femoris). 8. Cycling in supine position - test of hip flexors and knee extensors (quadriceps muscle). The feet are placed into a rotor, and the patient is trying to perform alternating pedaling movements. This could be performed first at 5 months postsurgery with the use of high load relief. In the period after the 12th month, the patient could cycle without any support. The left leg was the dominant one in this exercise. 9. Knee flexion in a prone position-test for the posterior group of LE muscles (semitendinosus, semimembranosus, biceps femoris, and gastrocnemius, innervated by the sacral spinal cord segments). There was no improvement in this muscle group.

\section{Neurophysiological Evaluation (Fig. 11, Table 5)}

During the first and second preoperative examinations, MEP and EMG studies detected efferent transmission from motor cortex centers to spinal cord motoneurons only to the level of innervation of the rectus abdominis muscles. Postoperatively, MEPs recorded from abdominal muscles on both sides were close to the normal recorded in healthy volunteers (more than $1,500 \mu \mathrm{V}$ ). At 5 months after the operation, excitation reached L1 to L4 spinal motoneurons followed by clear signs of recovery of contraction of rectus femoris muscles. An increase in the amplitudes in MEPs recorded in subsequent trials was prominent for the left rectus femoris muscle and reached $300 \mu \mathrm{V}$ at 14 months. MEPs from the right rectus femoris muscle were comparable to the one taken from the left rectus femoris at 5 months but decreased at 8 and 11 months and could not be recorded at 14 and 17 months. Activation of the corticospinal connections to L5-S1 motoneurons following the magnetic field excitation was only recorded rudimentarily from the distal muscles

Table 4. Summary of the Most Important Correlations Found During the Performed Tests of Neurological and Physiotherapeutic Achievement

\begin{tabular}{lcr}
\hline Variable & $\begin{array}{c}\text { Spearman } \\
\text { Coefficient }\end{array}$ & $p$ Level \\
\hline ASIA motor score-right and ASIA motor score-left & 0.83 & $<0.01$ \\
Leg adduction-left and leg adduction-right & 0.92 & $<0.01$ \\
ASIA Light Touch-left and ASIA Light Touch-right & 0.80 & $<0.01$ \\
ASIA Pin Prick-left and ASIA Pin Prick-right & 0.83 & $<0.01$ \\
ASIA motor score-left and Walking Index & 0.93 & $<0.01$ \\
ASIA motor score-right and Walking Index & 0.85 & $<0.01$ \\
ASIA Light Touch-right and Walking Index & 0.68 & 0.05 \\
ASIA Pin Prick-right and Walking Index & 0.71 & 0.03 \\
FIM and Walking Index & 0.93 & $<0.01$ \\
Leg adduction-right and Ashworth spasticity-right & -0.72 & 0.03 \\
Leg adduction-left and Ashworth spasticity-left & -0.73 & 0.02 \\
\hline
\end{tabular}

ASIA, American Spinal Cord Injury Association; FIM, Functional Independence Measure. 
PREOPERATIVE RECORDINGS

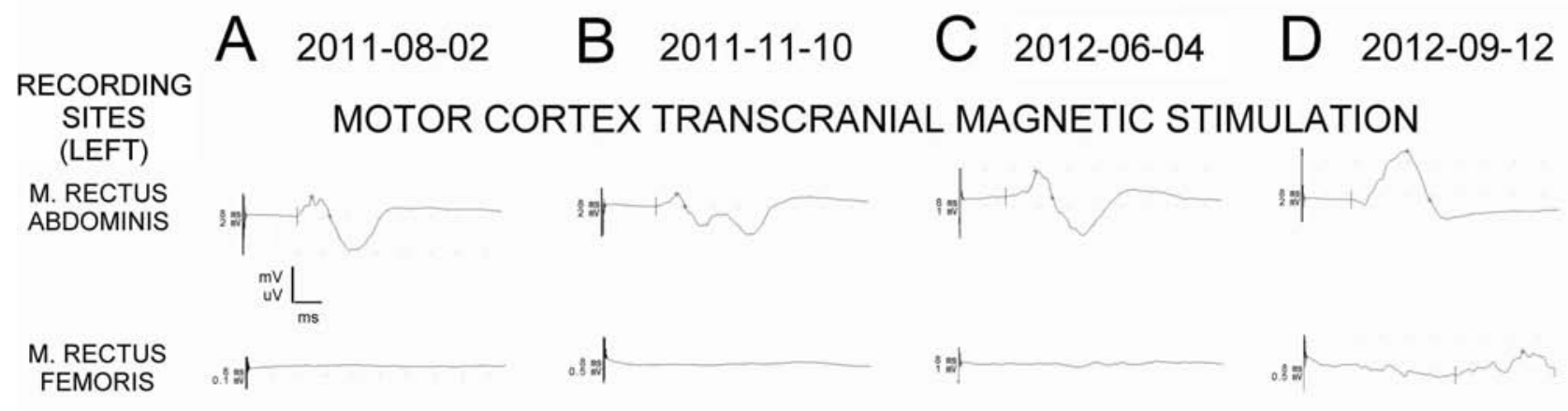

EMG RECORDINGS (ATTEMPT OF MAXIMAL CONTRACTION)

M. RECTUS ABDOMINIS
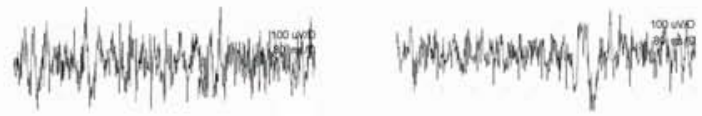

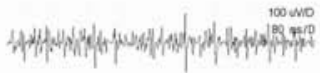

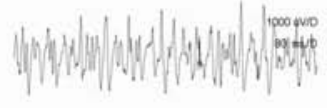

M. RECTUS

FEMORIS

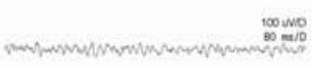

$\lim _{\substack{\infty \\ \text { sims } \\ 0 \rightarrow 0}}$

Whendy

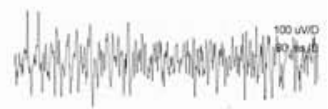

M. EXTENSOR DIGITI

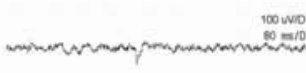

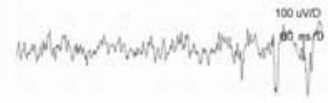

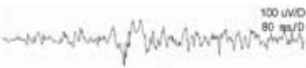

POSTOPERATIVE RECORDINGS

\section{E 2012-12-13 F 2013-03-27 G 2013-07-03 H 2013-10-02}

\section{MOTOR CORTEX TRANSCRANIAL MAGNETIC STIMULATION}

M. RECTUS ABDOMINIS

M. RECTUS FEMORIS
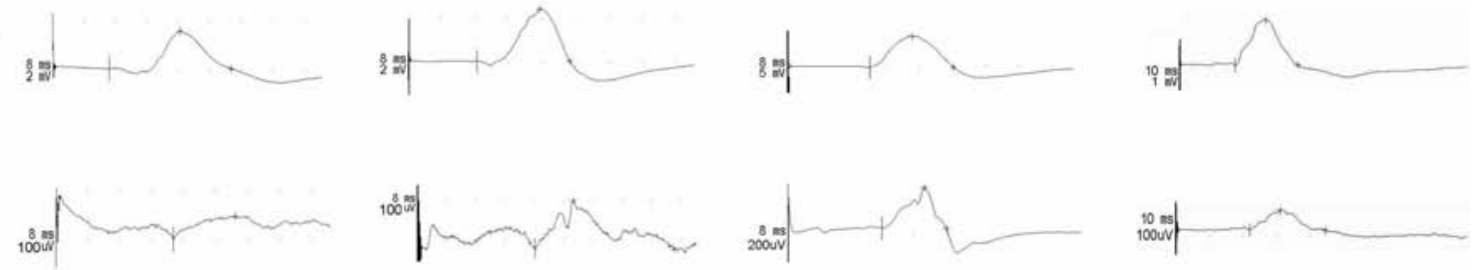

\section{EMG RECORDINGS (ATTEMPT OF MAXIMAL CONTRACTION)}

M. RECTUS
ABDOMINIS
M. RECTUS
FEMORIS
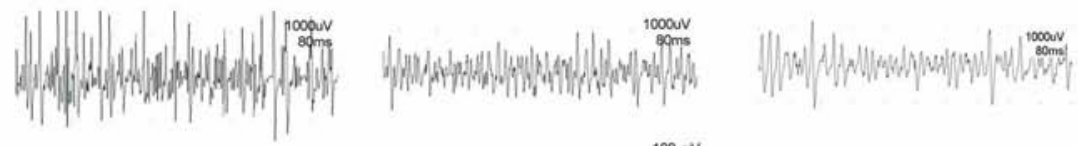

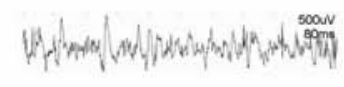

M. EXTENSOR
DIGITI
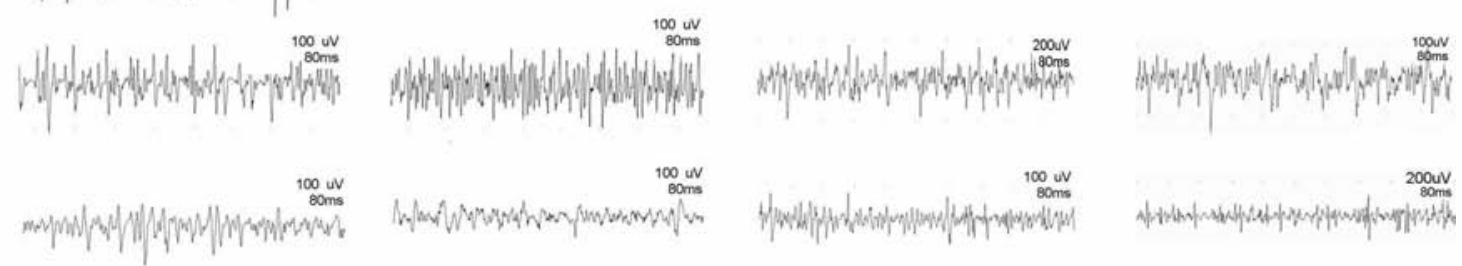

M.

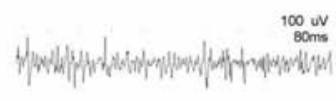

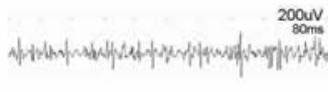

Figure 11. Pre- and postoperative comparison of MEPs and EMG recordings. Comparison of MEP recordings (upper traces) and EMG recordings (lower traces) performed pre- (A, B) and postoperatively (C, 1 month postsurgery, D, 5 months, E, 8 months, F, 11 months, G, 14 months, H, 17 months). Recordings were performed from the left LE. Note different amplifications of recordings and increase in MEP amplitudes recorded from both rectus abdominis and rectus femoris muscles (spinal efferent transmission recovery) as well as the improvement of motor unit activity in rectus abdominis and rectus femoris muscles observed in EMG recordings. The calibration bars of amplification (vertical, in millivolts or microvolts) and time base (horizontal, in milliseconds) for all MEP and EMG recordings are shown in (A). 


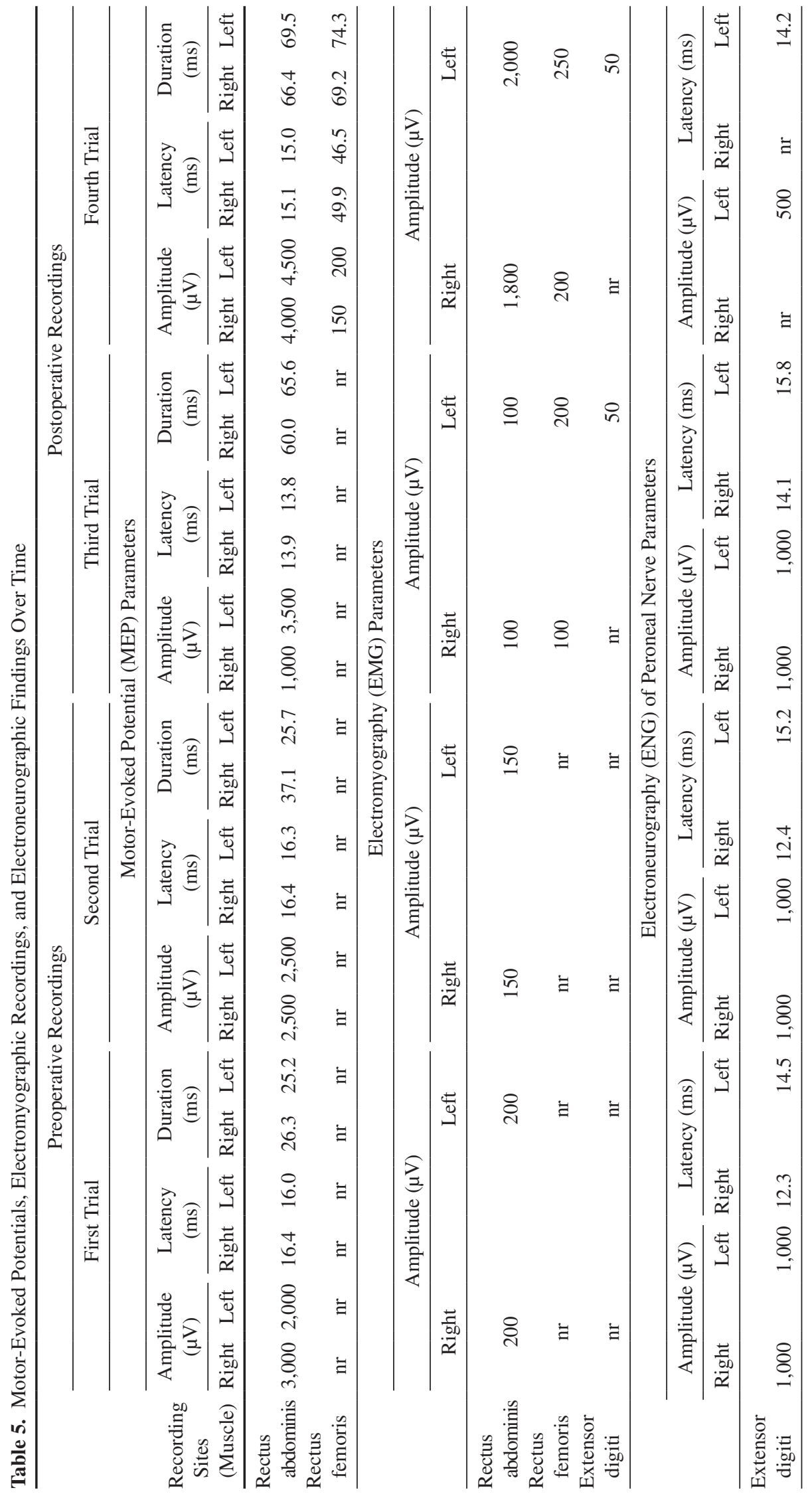




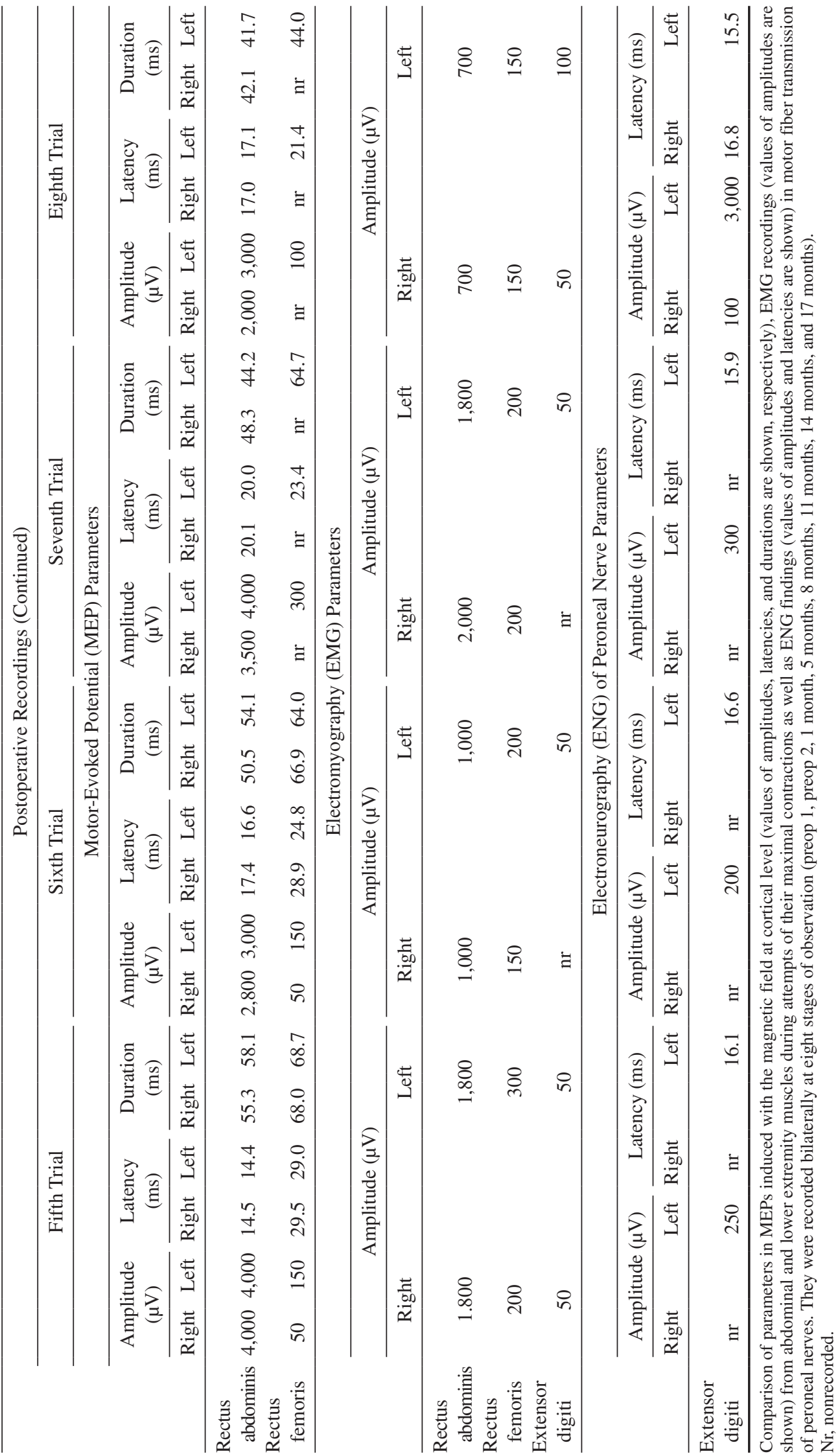


of the left LE. The first observed low-amplitude MEP recordings were associated with an increased latency and duration of potentials, but a tendency for shortening was detected during recordings at 11, 14, and 17 months (Fig. 12). Postoperative EMG recordings from LE showed voluntary muscle contractions recorded as action potentials from the rectus femoris muscles and the left digital extensor during the attempt of maximal muscle contraction, which were not present preoperatively. EMG recordings (both surface and needle performed) confirmed the voluntary contraction of muscles when recorded mainly on the left side. The frequency of positive EMG recordings in the proximal LE muscles was higher on the left side. Low frequency of the positive EMG recordings from distal muscles of the left LE, as well as absent or very weak responses, were found for the distal muscles of the right LE. ENG studies suggested this was related to degenerative changes in the peripheral motor fibers in the peroneal nerves. ENG studies showed their axonal type basing on recorded low amplitudes of M-wave potentials. Needle EMG recordings performed from active muscles during voluntary contraction showed at rest also positive sharp potentials in almost all of the recordings (20/24 trials), indicating a degenerative process at the neuromuscular junctions. The above observations were confirmed by three independent neurophysiologists involved in the neurophysiological tests.
Urodynamic studies did not show any difference in control examinations. A hyper-reflexia of the bladder detrusor and dyssynergy between the bladder detrusor and urethral sphincter activity was noted both pre- and postoperatively. There was no EMG evidence of voluntary functional activation of the anal sphincter. However, an improvement of bladder sensation helped the patient determine the timing for voiding. After surgery, the patient reported that he had regained the ability to obtain and maintain erection without the need for pharmacological support.

\section{Psychological Evaluation}

Clinical observation performed for 19 months after the operative treatment revealed positive changes in the psychological and social profile of the patient. This included a change of the style of interpersonal relations from passive (before treatment) to dominant (increase of MMPI-2 Leary index from 4 to 7), a better resolution of anger and aggression (increase of Leary index from 4 to 7), a decrease in anxiety level (EPQ-R: a decrease from 8 sten to 5 sten), an increase in the sense of self-control and efficacy in life (GSES: increase from 5 sten to 7 sten), and an increased satisfaction in interpersonal relations (including sexual). Negative observations concerned the patient's lower threshold of tolerance of frustration and higher irritability. Conclusively, an improvement of the

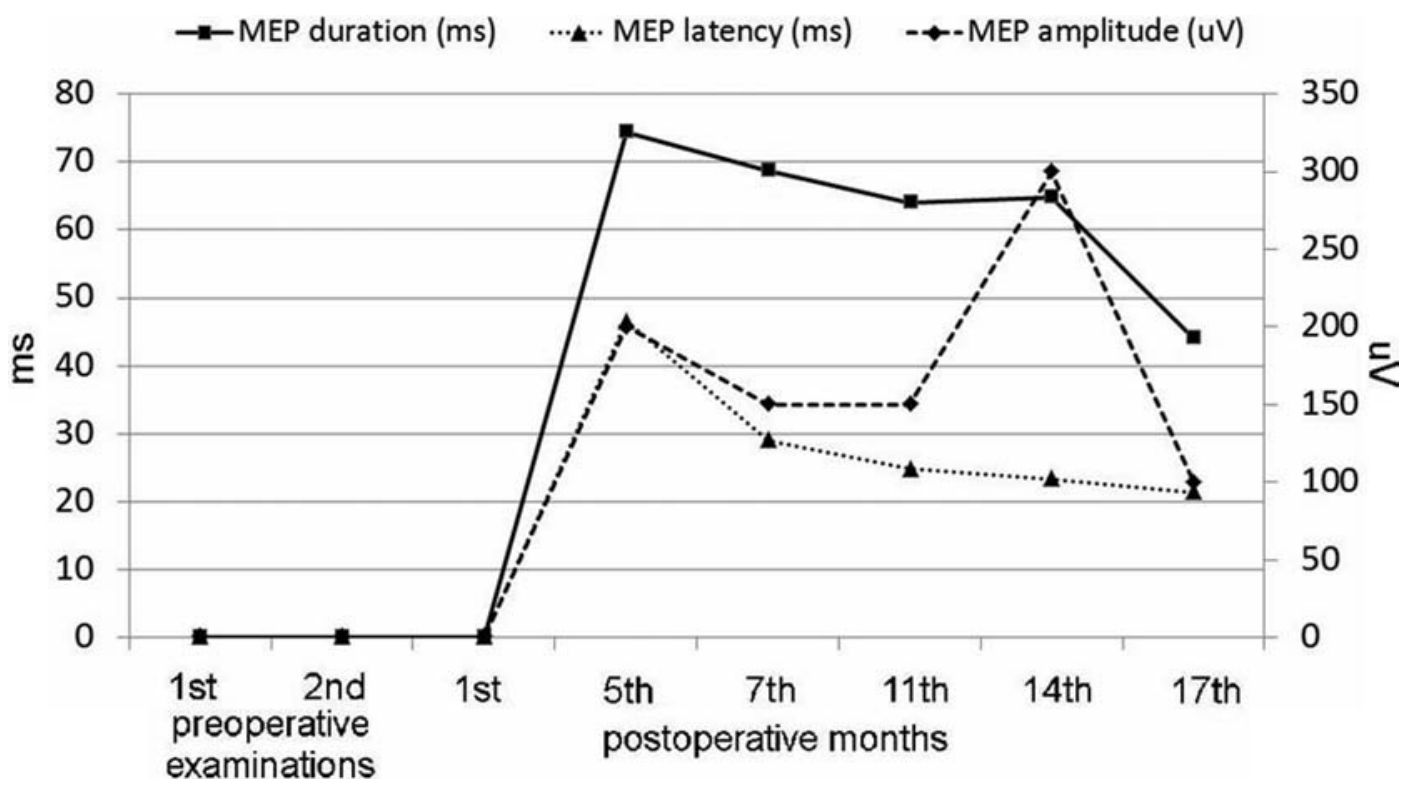

Figure 12. Variability of the MEP duration, latency, and amplitude parameters, recorded at stages during the neurophysiological assessment of the left rectus femoris muscle. There was no muscle response to motor cortex activation preoperatively. Note the continued muscle response after the fifth postoperative month and the improvement in spinal cord efferent transmission, registered as a gradual decrease of the latency and duration of the potential. A smaller MEP response of the right rectus femoris muscle (shown only numerically in Table 5) was present at 5-11 months but was not observed in the following two trials. 
mental state of the patient was noted, especially with respect to satisfying the most important needs like the sense of safety, social acceptability, and quality of life. According to the patient, his improved quality of life was influenced by both the neurological improvement, giving him a higher degree of independence, and the fact of participation in the therapeutic project.

\section{DISCUSSION}

\section{Safety of the Experimental Procedure}

The operation of the craniotomy for unilateral bulbectomy, followed by isolation and culture of OECs and ONFs and their transplantation into the spinal cord with simultaneous bridging of the gap with autologous sural nerves, was safe over the period of 19 months of postsurgical observation. There was no general and neurological deterioration, neuropathic pain, infection, spine instability, stenosis, tumors, or progressive myelopathy in all observational periods. Sterility tests of cell cultures and the CSF did not show any bacterial or fungal infection. The implanted nerve strips retained their structure and integrated well with the host spinal cord tissue, making efficient contact with the sectioned cord stumps. MRI revealed both pre- and postoperatively the development of small areas of myelopathy in the spinal cord adjacent to the lesion area. They remained radiologically stabile and did not influence the neurological and neurophysiological state of the patient (Fig. 5). We used measurements of the FA to assess the degree of integrity of spinal cord white matter tracts in the spinal cord (Table 2) (40). Values of FA measured at two levels above and below the lesion focus were typical for cases of spinal cord lesion $(29,41)$ and did not deteriorate in the subsequent studies, which confirms the assumption that the observed spinal cord degeneration was not progressive. Five weeks postsurgery, a DTI study showed a realignment of the tracts of water diffusion through the area of spinal cord reconstruction, followed in the next studies by a gap of water diffusion, as in the preoperative studies (Fig. 6). The early realignment of the tracts in DTI is unlikely to be indicative of fiber regeneration because this process would be expected to need longer periods of time. It rather showed a good integrity of degenerated spinal cord and nerve endoneural channels. The gap of water diffusion, occurring in the later DTI studies, was not associated with any worsening of the patient's neurophysiological and neurological state, and conventional MRI images showed that the nerve implants remained well integrated with the host spinal cord tissue. We believe that either the performed study was not sensitive enough to show thin regenerating fibers crossing the implant, or the pattern of fiber regrowth might not have been longitudinal and changed the direction of water diffusion in the reconstructed area.
Unexpectedly, the patient regained some smell perception on the side of the bulbectomy 3 weeks after the craniotomy. Our first explanation was that following the sustained operation of anterofrontoethmoid sphenoidectomy, new anatomical connections between the left and right nostrils for the airway passage could have been created (as in septostomy, etc.), enabling the right $\mathrm{OB}$ to be excited by the odor stimuli entering the left nostril. Yet CT scans and laryngological fiberoscopy could not confirm this hypothesis. A possibility of an efficient bilateral flow of the air through the posterior nares was also not confirmed. For example, we noted in some of our patients treated for anterior skull base fracture, in whom the lesioned OB had to be removed, that they did not regain olfaction on the side of the bulbectomy (unpublished data). As those patients underwent cranialization of the skull base with the use of a periosteal flap that separated the fila olfactoria from the olfactory cortex, and our patient did not, we suggest that a plastic response or a regenerative response within the olfactory system may have occurred in the present case. Although the phenomenon of direct reinnervation of the olfactory cortex by olfactory axons, after bulbectomy, has been described in mammals (15), further investigation of our observation is required and is beyond the scope of this article.

\section{The Pattern of Neurological Recovery: Spontaneous Versus Induced Recovery?}

In this article, we show an essential neurological recovery of an ASIA A patient with chronic 21-month paraplegia to ASIA C grade (sensory and motor incomplete) within a period of 19 months after an operative intervention consisting of spinal cord scar resection, intraparenchymal bulbar cell microinjection, and reconnection of the spinal cord stumps with sural nerve grafts. The first issue that has to be discussed is whether the observed recovery could have been spontaneous and triggered by rehabilitation rather than a result of the transplantation procedure. Preoperative MRI images, as well as intraoperative exploration, showed an almost total physical disconnection of the spinal cord stumps at upper vertebral level Th9. Only a thin 2-mm-thick spur of tissue was connecting the stumps (Figs. 1 and 3). Serial preoperative MEP and EMG studies indicated that this tissue was not conducting electrical stimuli to the lumbar group of motoneurons below the area of injury. We did not remove it because we believed that it may be able to act as a scaffold to guide new regrowing axons and also might help to mechanically stabilize our grafted nerve strips.

The preoperative clinical, electrophysiological, radiological, and intraoperative data showed that the knife entry produced a rare type of severe spinal cord lesion seen in 
humans, resembling an experimental situation of an isolated spinal cord transection with minimal involvement of vertebral structures. In our opinion, the prognostic signs made spontaneous recovery unlikely. A panel of leading experts in the field of treatment of SCIs, who analyzed the data from three large double-blind placebo-controlled trials on neuroprotection in SCI-the National Acute Spinal Cord Injury Study (NASCIS), Sygen (monosialotetrahexosylganglioside; GM ganglioside), and GK-11 (gacyclidine) trials-concluded that a spontaneous recovery of motor and sensory in an ASIA A patient may be possible in $20 \%$ of the cases in the first year after injury but mainly in the first 3 months (13). Another study showed that most spontaneous motor recovery in ASIA A patients occurred in the first 6 months after injury and was almost absent after 12 months (45). Additional data on the late spontaneous recovery of 987 SCI patients showed that between the first and fifth year after injury, only $5.6 \%$ of the ASIA A patients may recover, from which $3.5 \%$ recover to ASIA B grade and only $1.05 \%$ to ASIA C and $1.05 \%$ to ASIA D (21). The data of Kirshblum et al. (21) are strong, even though they were not supported by electrophysiological studies and information about how the patients were rehabilitated. To be sure that our patient would not improve spontaneously, and to exclude the "nocebo" effect seen in inappropriately rehabilitated patients, he underwent an additional 8-month intense preoperative rehabilitation program in one of the Polish reference centers for rehabilitation of SCI patients. MEP and EMG studies performed at the beginning and end of this 8-month training, together with regular neurological assessments, did not show neurological recovery. For this reason, we consider that the probability of spontaneous recovery in our patient was lower than $1 \%$.

We observed a gradual recovery of both sensory and motor function that started after the fourth month postoperatively. This recovery was nonlinear, having two critical periods: the fifth through sixth months, when the first evident signs of sacral sparing and voluntary muscle contractions from the first motor segments below the level of injury appeared (the patient became ASIA B), and the period after the 10th month, when the patient turned to ASIA C. The motor recovery occurred in a segmental pattern, starting at 5 months from an increase of the strength of the lower abdominal muscles and other trunk muscles (lower thoracic motor segments), followed by a gradual increase of voluntary LE adduction (L1 segments), reaching 3 points according to the MRC scale in the left LE and 2 points in the right LE. The voluntary control of the musculature proceeded in time downward, predominantly to the left LE, and included hip flexion (L2 segments, 1 point according to MRC) and knee extension (L3 segments, 2 points according to the MRC scale) (Fig. 9). This motor recovery was preceded by a marked increase in muscle mass in the left thigh as a sign of ongoing reinnervation. The recovery concerned not only the ZPP but also several motor segments below this zone and has been considered to be a sign of spinal cord repair (13). The observed appearance of new voluntary muscle function, coming from reinnervated motor segments below the level of spinal cord transection, could also be confirmed more objectively by the performance of MEP and EMG studies. Repeated electrophysiological studies were predictive for the observed motor recovery in the ASIA motor investigation and indicated again a dominant motor recovery in the left LE up to the L5-S1 segments (Fig. 11 and Table 5). Difficulties in MEP recordings from the proximal muscles of the right LE, the lack of MEP recordings and low amplitudes of the EMG response in its distal muscles, and the worsening of the ENG values in the right peroneal nerve (Table 5) may be explained by an ongoing peripheral nerve degeneration of central origin (33). This phenomenon was described in patients after stroke (16) and does not arise from a direct lesion of the peripheral nerves, but from a long-term lack of activation of spinal cord motoneurons from neurons of supraspinal origin. In our case, such lack of activation or weak activation may have occurred in the motoneurons innervating the right LE.

The improvement in efferent spinal cord transmission was characterized by increasing MEP amplitudes in the abdominal muscles and mainly in the left LE muscles and also as gradual increase in the velocity of nerve signal conduction, registered as shortening of the latency of signal conduction and the duration of motor potential (Table 5, Fig. 12). The first latency (46.5 ms) and duration of the MEP response $(74.3 \mathrm{~ms}$ ) of the left femoris muscle, registered at 5 months postoperatively, were pathologically protracted. With time, a gradual normalization of these parameters was observed. Hence, the latency of the MEP response at 14 and 17 months postsurgery reached values typical for the healthy population $(23.4 \mathrm{~ms}$ and $21 \mathrm{~ms}$, respectively). In conclusion, the improvement in efferent spinal cord transmission gave evidence of ongoing longdistance motor fiber regeneration (amplitude increase), possibly from the corticospinal tracts, and remyelination of part of these tracts (latency and potential duration normalization), occurring mainly from the part of the spinal cord that had been bridged with the nerve strips and was a good prognostic factor for the observed neurological improvement.

Starting from the fifth month after surgery, as in the case of the motor function tests, we observed a timely nonlinear pattern of recovery of superficial sensation, with higher scores achieved in the right LE both in the ASIA Light Touch and Pin Prick scores in dermatomes from L5 
to S5, depending on the type of tested modality (Fig. 8). This may be consistent with regeneration of the primary afferent fibers crossing the midline below the injury site and regenerating along the nerves implanted predominantly in the left half of the spinal cord as spinothalamic tracts. Additionally, the recovery of deep sensation on both sides, which was stronger in the feet, may be consistent with regeneration of primary afferent proprioceptive fibers from lower lumbar and sacral segments, ascending in the dorsal columns closest to the midline and crossing the bridged injury on both sides. The lesser recovery of motor function in the right LE and superficial sensation in the left LE might have been due to either plasticity of fibers that have regenerated across the nerve grafts on the left or minimal regrowth of fibers crossing the OECinfiltrated scar tissue on the right side of the spinal cord.

Taken together, all observed recovery of motor and sensory function had a partial Brown-Sequard pattern and fitted exactly with the location of the repair, suggesting that the bridge of peripheral nerve grafts that preferentially reconnected the left half and the medial part of the right half of the spinal cord stumps (Fig. 13). Additional clinical observations concerned the reappearance of the left Achilles tendon reflex and the disappearance of the Babinski sign. The observed normalization of reflex activity may also indicate an improved supraspinal control of local spinal cord neuronal circuits. We also noticed some symptoms of improved autonomic function, such as an improvement of bladder sensation, confirmed in urodynamic studies; an improvement of erection control without the need for pharmacological support; and improved vascular autoregulation mainly in the left LE.

The observed statistically significant improvement of sensorimotor function had a positive impact on the achievements in physical exercises during rehabilitation and significantly influenced the results from the FIM tests and the ability of the patient to walk, measured as increased WI (Table 4). The improved walking was not only due to the increasing strength of trunk and LE muscles but also to the recovery of deep sensation in both LEs and superficial sensation mainly in the right LE. The sensory recovery enabled better coordination and perception of leg movements and improved the quality of the walk.

\section{Aspects of the Operation Contributing to the Neurological Recovery}

Because our approach, as oriented to give the patient the "best medical treatment" was complex, it is difficult to determine which aspects of the interventions contributed to the observed neurological recovery. The surgical intervention included spinal cord untethering, resection of the intraparenchymal scar tissue, injection into the spinal cord stumps and the rim of spared tissue of a mixture of bulbar OECs/ONFs, and reconnection of the stumps with four strips of sural nerves and was followed by a long and intense neurorehabilitation program. Each single intervention had its importance but, in our opinion, could not be in itself sufficient if applied without the others.

The spinal cord untethering (myeloadhesiolysis) may have improved the vascular supply in the area of spinal cord reconstruction and could be beneficial for the integration of the nerve grafts with the spinal cord tissue. Studies on a large group of patients with SCI undergoing late myeloadhesiolysis did not show any significant influence of this intervention on the sensorimotor recovery (11). The removal of the intraparenchymal scar tissue was beneficial because it eliminated some of the physical and chemical barriers for axonal regeneration contained in the scar (12) and turned the chronic SCI into an acute one, enabling a better interaction of the transplanted OECs and ONFs with host astrocytes. We consider it most likely that the crucial interventions during the operation were the intraparenchymal transplantation of OECs/ONFs and the reconnection of the spinal cord stumps with strips of peripheral nerves. Transplanted bulbar cells could have been responsible for realignment of the astrocytic processes and "opened the door" for regrowth of central axons (24).

The specific clinical condition of our patient, suffering from a chronic inflammatory condition of the nasal mucosa, allowed us to use for the first time OECs isolated from the human OB. There is growing evidence in the literature that bulbar OECs have stronger regenerationpromoting capacity than mucosal OECs $(18,32,39,46)$. We also observed in our previous trial on ASIA A paraplegic patients that mucosal OECs/ONFs gave minimal neurological improvement in all operated patients (43).

The only completed phase I clinical study on application of purified OECs in the treatment of human paraplegia did not show any efficacy of transplanted purified autologous mucosal OECs (30). As in our previous study, we transplanted cultures containing mixtures of OECs and ONFs. The ONFs form an intimate outer cover on the outer surface of the OECs, with the nerve fibers on the inner surface. After transplantation into the corticospinal tract or optic nerve lesions, the advancing ONFs precede and establish a channel for the advance of the OECs $(23,25)$. In all these situations, OECs and ONFs seem to act together as essential and complementary components of a proregenerative tissue, and our experience (unpublished) is that purified OECs do not survive well after transplantation in rat spinal cord lesions. The mechanism of these complex interactions between OECs and ONFs is unknown. It certainly involves intimate surface-tosurface contact, implying a dependence on the interactions between membrane-bound molecules and leading to a 


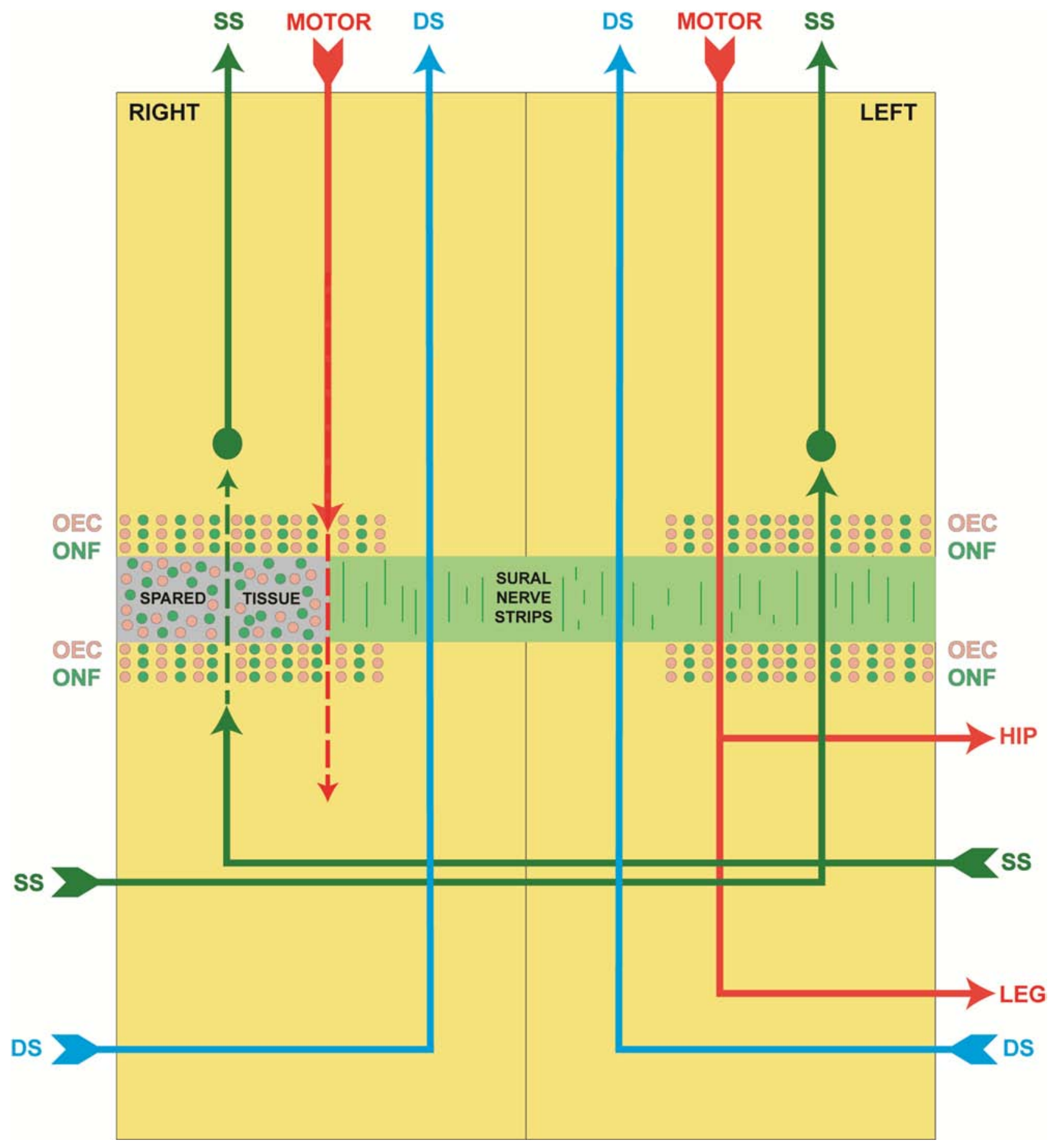


basal lamina forming on the surface of the OECs facing the ONFs.

The use of peripheral nerve grafts to bridge the sectioned spinal cord in experimental animals has been described three decades ago $(7,38)$. In these studies, only some populations of sensory neurons and intrinsic spinal cord neurons were seen to elongate their axons within the implants and enter, for short distances, the spinal cord. Newer modifications of the described methods claimed, both in preclinical and clinical studies, that if the nerve grafts connect the white matter above the level of injury with the gray matter below and the white matter below the injury with the gray matter above, with local administration of acidic fibroblast growth factor, a regrowth of longdistance supraspinal axons may be possible $(4,5)$ and may be beneficial for the patient $(5)$. Cheng et al. $(4,5)$ claimed that they rerouted the regenerative pathway from the nonpermissive white matter to the more permissive gray matter that is devoid of factors hindering regeneration.

In our technique, multiple microinjections of a mixture of OECs and ONFs into the lateral columns of the spinal cord may have enabled the necessary interaction with host astrocytes to make possible the regeneration of both efferent and afferent axons throughout the bridged nerves into the caudal and rostral stumps. The nerve strips were placed along the long axis of both white and gray matter and enabled the central axons to grow within their natural anatomical compartments. Implanted peripheral nerve grafts served as guidance tubes with the appropriate extracellular environment for axon adhesion and elongation and also as a large source of Schwann cells, known to be able to myelinate fibers of central origin and to secrete a variety of neurotrophic factors (1). The administration of methylprednisolone may have led to an increase in the number of myelinated CNS axons growing through the implants and also may have given better integration of the transplant with the host tissue as described by Chen et al. (3). Taken together, our experimental approach of combining Schwann cell bridges and bulbar OECs was very similar to the one described in rats by Ramon-Cueto et al. (37). In that study, the authors demonstrated that supraspinal axons regenerating through the transplant reentered the spinal cord and made synaptic connections. Thus, we believe that the neurological recovery in our patient indicates regeneration of central nervous system fibers that crossed the host/peripheral nerve interfaces and grew for a considerable distance to make functional connections in the cord (Fig. 13).

\section{CONCLUSION}

The results from the treatment of the first patient with a complete SCI receiving transplantation of bulbar OECs/ ONFs and simultaneous reconstruction of the spinal cord gap with peripheral nerve implants are very encouraging but have to be confirmed in a larger group of patients sustaining similar types of SCI. Further laboratory studies will be needed to elucidate the properties of human bulbar OECs/ONFs and their interaction with peripheral nerve bridges or artificial implants in vitro as well as their reparative potential in vivo. We are investigating surgical techniques for minimally invasive access to the human OB. There remains a possibility that sources of other, more readily obtainable, reparative cells may be discovered.

\section{FACING PAGE}

Figure 13. Schematic diagram showing the proposed pattern of fiber regeneration. The injury consisted of a complete transection with 8-mm separation over the left three fourths of the spinal cord at the vertebral T9 level, resulting in complete loss of motor and sensory function from and including the spinal L1 segment downward. Cultured OECs/ONFs were microinjected into the lateral parts of the upper and lower stumps adjacent to the injury and into the rim of nonfunctional spared tissue on the right. Four strips of sural nerve (green) were used to bridge the gap between the stumps over the remaining three fourths of the cord. Within 5 months, motor recovery (red) had started around the left hip (L1/2 cord segments) and superficial sensation (SS, green) over dermatomal levels S3-5 (sacral sparing). By 12 months, motor recovery on the left had extended to the leg (L2,3), and deep sensation (DS, blue) had returned on both sides down to the feet (L5/S1). On the right side of the body, there was a slight but much inferior and incomplete recovery of motor control, while there was a substantial recovery of SS. In contrast, on the left, there was a predominant motor recovery but minimal recovery of SS. The patient has been restored from complete paraplegia to a condition resembling an incomplete Brown-Sequard syndrome. Together with the neurophysiological data, this is consistent with regeneration of descending motor control (corticospinal) fibers (red) across the injury and their progressive descent with time on the left side of the distal cord. Similarly, the primary fibers carrying SS (green) from the right side of the body, which cross below the injury level, have also regenerated across the injury site and made contact with local spinothalamic relay neurons on the left side of the cord above the injury. The fibers of DS (blue) seem to have been able to cross the medial cord regions (the dorsal columns), which were bridged by sural nerve strips alone, but in the absence of local relay neurons in the cord, the DS fibers require a longer time for the greater distance of regeneration needed for sensation to reach the brain. Unlike the progressive downward extension of the motor fibers, the ascending sensory fibers from all caudal levels are severed at the level of the injury, and there is no preferential recovery of upper versus lower segmental fibers. The ability of motor and SS fibers to cross the injury requires both OECs/ONFs and sural nerve bridging. There is an indication that DS fibers may have regenerated across sural nerve bridges without OECs/ONFs. Infiltrating the nonfunctional residual tissue on the right of the cord with OECs/ONFs, but without interposed peripheral nerve tissue, gives a much inferior motor and SS result. 
ACKNOWLEDGMENTS: The authors are very grateful to Dr. Dariusz Szarek from the Department of Neurosurgery Wroclaw Medical University for the help during the operation of OEC transplantation and to Dr. Krzysztof Fortuna for the performed statistical analysis of the results. This work was supported by funds from the Wroclaw Medical University in the years 20092012 (study number ST 406) and the Nicholls Spinal Injury Foundation and the UK Stem Cell Foundation. The authors declare no conflict of interest.

\section{REFERENCES}

1. Bunge, M. B. Bridging the transected or contused adult rat spinal cord with Schwann cell and olfactory ensheathing glia transplants. Prog. Brain Res. 137:275-282; 2002.

2. Butcher, J. N.; Graham, J. R.; Ben-Porath, Y. S.; Tellegen, A.; Grant, W.; Dahlstrom, W. G.; Kaemmer, B. The Minnesota Multiphasic Personality Inventory (MMPI-2). Minneapolis, MN: University of Minnesota Press; 2001.

3. Chen, A.; Xu, X. M.; Kleitman, N.; Bunge, M. B. Methylprednisolone administration improves axonal regeneration into Schwann cell grafts in transected adult rat thoracic spinal cord. Exp. Neurol. 138(2):261-276; 1996.

4. Cheng, H.; Cao, Y.; Olson, L. Spinal cord repair in adult paraplegic rats: Partial restoration of hind limb function. Science 273(5274):510-513; 1996.

5. Cheng, H.; Liao, K. K.; Liao, S. F.; Chuang, T. Y.; Shih, Y. H. Spinal cord repair with acidic fibroblast growth factor as a treatment for a patient with chronic paraplegia. Spine 29(14):E284-288; 2004

6. Chhabra, H. S.; Lima, C.; Sachdeva, S.; Mittal, A.; Nigam, V.; Chaturvedi, D.; Arora, M.; Aggarwal, A.; Kapur, R.; Khan, T. A. Autologous olfactory mucosal transplant in chronic spinal cord injury: An Indian Pilot Study. Spinal Cord 47(12):887-895; 2009.

7. David, S.; Aguayo, A. J. Axonal elongation into peripheral nervous system "bridges" after central nervous system injury in adult rats. Science 214(4523):931-933; 1981.

8. Ditunno, Jr., J. F.; Ditunno, P. L.; Scivoletto, G.; Patrick, M.; Dijkers, M.; Barbeau, H.; Burns, A. S.; Marino, R. J.; Schmidt-Read, M. The Walking Index for Spinal Cord Injury (WISCI/WISCI II): Nature, metric properties, use and misuse. Spinal Cord 51(5):346-355; 2013.

9. Doucette, J. R. The glial cells in the nerve fiber layer of the rat olfactory bulb. Anat. Rec. 210(2):385-391; 1984.

10. Eyseneck, H. J.; Eyseneck, S. B. G. Manual of the Eyseneck Personality Questionnaire. London, UK: Hodder and Stoughton; 1975.

11. Falci, S. P.; Indeck, C.; Lammertse, D. P. Posttraumatic spinal cord tethering and syringomyelia: Surgical treatment and long-term outcome. J. Neurosurg. Spine 11(4):445460; 2009

12. Fawcett, J. W.; Asher, R. A. The glial scar and central nervous system repair. Brain Res. Bull. 49(6):377-391; 1999.

13. Fawcett, J. W.; Curt, A.; Steeves, J. D.; Coleman, W. P.; Tuszynski, M. H.; Lammertse, D.; Bartlett, P. F.; Blight, A. R.; Dietz, V.; Ditunno, J.; Dobkin, B. H.; Havton, L. A.; Ellaway, P. H.; Fehlings, M. G.; Privat, A.; Grossman, R.; Guest, J. D.; Kleitman, N.; Nakamura, M.; Gaviria, M.; Short, D. Guidelines for the conduct of clinical trials for spinal cord injury as developed by the ICCP panel: Spontaneous recovery after spinal cord injury and statistical power needed for therapeutic clinical trials. Spinal Cord 45(3):190-205; 2007.
14. Feron, F.; Perry, C.; Cochrane, J.; Licina, P.; Nowitzke, A.; Urquhart, S.; Geraghty, T.; Mackay-Sim, A. Autologous olfactory ensheathing cell transplantation in human spinal cord injury. Brain 128(Pt 12):2951-2960; 2005.

15. Graziadei, P. P.; Levine, R. R.; Graziadei, G. A. Regeneration of olfactory axons and synapse formation in the forebrain after bulbectomy in neonatal mice. Proc. Natl. Acad. Sci. USA 75(10):5230-5234; 1978 .

16. Hallet, M.; Chokroverty, S. Magnetic stimulation in clinical neurophysiology: Philadelphia PA: Elsevier; 2005.

17. Halon, A.; Materna, V.; Drag-Zalesinska, M.; NowakMarkwitz, E.; Gansukh, T.; Donizy, P.; Spaczynski, M.; Zabel, M.; Dietel, M.; Lage, H.; Surowiak P. Estrogen receptor alpha expression in ovarian cancer predicts longer overall survival. Pathol. Oncol. Res. 17(3):511-518; 2011.

18. Ibrahim, A.; Li, D.; Collins, A.; Tabakow, P.; Raisman, G.; Li, Y. Comparison of olfactory bulbar and mucosal cultures in a rat rhizotomy model. Cell Transplant. 23(11):1465$1470 ; 2014$.

19. Jerusalem, M.; Schwarzer, R. Generalized Self-Efficacy Scale GSE. Berlin, Germany: Freie Universitat; 1993.

20. Keyvan-Fouladi, N.; Raisman, G.; Li, Y. Functional repair of the corticospinal tract by delayed transplantation of olfactory ensheathing cells in adult rats. J. Neurosci. 23(28):9428-9434; 2003.

21. Kirshblum, S.; Millis, S.; McKinley, W.; Tulsky, D. Late neurologic recovery after traumatic spinal cord injury. Arch. Phys. Med. Rehabil. 85(11):1811-1817; 2004.

22. Li, Y.; Decherchi, P.; Raisman, G. Transplantation of olfactory ensheathing cells into spinal cord lesions restores breathing and climbing. J. Neurosci. 23(3):727-731; 2003.

23. Li, Y.; Field, P. M.; Raisman, G. Regeneration of adult rat corticospinal axons induced by transplanted olfactory ensheathing cells. J. Neurosci. 18(24):10514-10524; 1998.

24. Li, Y.; Li, D.; Raisman, G. Interaction of olfactory ensheathing cells with astrocytes may be the key to repair of tract injuries in the spinal cord: The 'pathway hypothesis'. J. Neurocytol. 34:343-351; 2005.

25. Li, Y.; Sauve, Y.; Li, D.; Lund, R. D.; Raisman, G. Transplanted olfactory ensheathing cells promote regeneration of cut adult rat optic nerve axons. J. Neurosci. 23(21):7783-7788; 2003.

26. Lima, C.; Escada, P.; Pratas-Vital, J.; Branco, C.; Arcangeli, C. A.; Lazzeri, G.; Maia, C. A.; Capucho, C.; HasseFerreira, A.; Peduzzi, J. D. Olfactory mucosal autografts and rehabilitation for chronic traumatic spinal cord injury. Neurorehabil. Neural Repair 24(1):10-22; 2010.

27. Lima, C.; Pratas-Vital, J.; Escada, P.; Hasse-Ferreira, A.; Capucho, C.; Peduzzi, J. D. Olfactory mucosa autografts in human spinal cord injury: A pilot clinical study. J. Spinal Cord Med. 29(3):191-203; discussion 204-206; 2006.

28. Lindsay, S. L.; Riddell, J. S.; Barnett, S. C. Olfactory mucosa for transplant-mediated repair: A complex tissue for a complex injury? Glia 58(2):125-134; 2010.

29. Loy, D. N.; Kim, J. H.; Xie, M.; Schmidt, R. E.; Trinkaus, K.; Song, S. K. Diffusion tensor imaging predicts hyperacute spinal cord injury severity. J. Neurotrauma 24(6):979_ 990; 2007.

30. Mackay-Sim, A.; Feron, F.; Cochrane, J.; Bassingthwaighte, L.; Bayliss, C.; Davies, W.; Fronek, P.; Gray, C.; Kerr, G.; Licina, P.; Nowitzke, A.; Perry, C.; Silburn, P. A.; Urquhart, S.; Geraghty, T. Autologous olfactory ensheathing cell transplantation in human paraplegia: A 3-year clinical trial. Brain 131(Pt9):2376-2386; 2008. 
31. Miedzybrodzki, R.; Tabakow, P.; Fortuna, W.; Czapiga, B.; Jarmundowicz, W. The olfactory bulb and olfactory mucosa obtained from human cadaver donors as a source of olfactory ensheathing cells. Glia 54(6):557-565; 2006.

32. Paviot, A.; Guerout, N.; Bon-Mardion, N.; Duclos, C.; Jean, L.; Boyer, O.; Marie, J. P. Efficiency of laryngeal motor nerve repair is greater with bulbar than with mucosal olfactory ensheathing cells. Neurobiol. Dis. 41(3):688-694; 2011.

33. Preston, D. C.; Shapiro, B. E. Electromyography and Neuromuscular Disorders, Clinical-Electrophysiologic Correlations, 2nd ed. Philadelphia, PA: Elsevier; 2005.

34. Raisman, G. Specialized neuroglial arrangement may explain the capacity of vomeronasal axons to reinnervate central neurons. Neuroscience 14(1):237-254; 1985.

35. Ramon-Cueto, A.; Cordero, M. I.; Santos-Benito, F. F.; Avila, J. Functional recovery of paraplegic rats and motor axon regeneration in their spinal cords by olfactory ensheathing glia. Neuron 25(2):425-435; 2000.

36. Ramon-Cueto, A.; Nieto-Sampedro, M. Glial cells from adult rat olfactory bulb: Immunocytochemical properties of pure cultures of ensheathing cells. Neuroscience 47(1): 213-220; 1992.

37. Ramon-Cueto, A.; Plant, G. W.; Avila, J.; Bunge, M. B. Long-distance axonal regeneration in the transected adult rat spinal cord is promoted by olfactory ensheathing glia transplants. J. Neurosci. 18(10):3803-3815; 1998.

38. Richardson, P. M.; McGuinness, U. M.; Aguayo, A. J. Peripheral nerve autografts to the rat spinal cord: Studies with axonal tracing methods. Brain Res. 237(1):147-162; 1982.

39. Santos-Benito, F. F.; Ramon-Cueto, A. Olfactory ensheathing glia transplantation: A therapy to promote repair in the mammalian central nervous system. Anat. Rec. B New Anat. 271(1):77-85; 2003.

40. Schwartz, E. D.; Chin, C. L.; Shumsky, J. S.; Jawad, A. F.; Brown, B. K.; Wehrli, S.; Tessler, A.; Murray, M.; Hackney, D. B. Apparent diffusion coefficients in spinal cord transplants and surrounding white matter correlate with degree of axonal dieback after injury in rats. AJNR Am. J. Neuroradiol. 26(1):7-18; 2005.

41. Shanmuganathan, K.; Gullapalli, R. P.; Zhuo, J.; Mirvis, S. E. Diffusion tensor MR imaging in cervical spine trauma. AJNR Am. J. Neuroradiol. 29(4):655-659; 2008.

42. Spielberger, C. D.; Gorusch, R. L.; Lushene, R. E. Manual for the State-Trait Anxiety Inventory. Mountain View, CA: Consulting Psychologists Press; 1970.

43. Tabakow, P.; Jarmundowicz, W.; Czapiga, B.; Fortuna, W.; Miedzybrodzki, R.; Czyz, M.; Huber, J.; Szarek, D.; Okurowski, S.; Szewczyk, P.; Gorski, A.; Raisman, G. Transplantation of autologous olfactory ensheathing cells in complete human spinal cord injury. Cell Transplant. 22(9):1591-1612; 2013.

44. van Mastrigt, R.; Griffiths, D. J. ICS standard for digital exchange of urodynamic study data. Neurourol. Urodyn. 23(3):280-281; 2004

45. Waters, R. L.; Adkins, R. H.; Yakura, J. S.; Sie, I. Motor and sensory recovery following complete tetraplegia. Arch. Phys. Med. Rehabil. 74(3):242-247; 1993.

46. Yamamoto, M.; Raisman, G.; Li, D.; Li, Y. Transplanted olfactory mucosal cells restore paw reaching function without regeneration of severed corticospinal tract fibres across the lesion. Brain Res. 1303:26-31; 2009. 